\title{
BMAL1 attenuates intracerebral hemorrhage-induced secondary brain injury in rats by regulating the Nrf2 signaling pathway
}

\author{
Yan Gong", Guoguo Zhang*, Bing Li, Cheng Cao, Demao Cao, Xiang Li, Haiying Li, Ming Ye, \\ Haitao Shen, Gang Chen
}

Department of Neurosurgery\& Brain and Nerve Research Laboratory, The First Affiliated Hospital of Soochow University, Suzhou, China

Contributions: (I) Conception and design: Y Gong, G Zhang, M Ye, H Shen; (II) Administrative support: M Ye, H Shen, G Chen; (III) Provision of study materials: M Ye, H Shen; (IV) Collection and assembly of data: C Cao, B Li, D Cao, X Li, H Li; (V) Data analysis and interpretation: Y Gong, G Zhang, X Li, H Li; (VI) Manuscript writing: All authors; (VII) Final approval of manuscript: All authors.

\#These authors contributed equally to this work.

Correspondence to: Ming Ye; Haitao Shen. Department of Neurosurgery\& Brain and Nerve Research Laboratory, The First Affiliated Hospital of Soochow University, 188 Shizi Street, Suzhou 215006, China. Email: yeming@suda.edu.com; dagezi120@126.com.

Background: Intracerebral hemorrhage (ICH) is a severe cerebrovascular disease with high morbidity and mortality rates. Oxidative stress and inflammation are important pathological mechanisms of secondary brain injury (SBI) after ICH. Brain and muscle Arnt-like protein 1 (BMAL1), which forms the core component of the circadian clock, was previously shown to be involved in many diseases and to participate in oxidative stress and inflammatory responses. However, the role of BMAL1 in SBI following ICH is unknown. In addition, treatments targeting miR-155 and its downstream signaling pathway may exert a beneficial effect on SBI after ICH, while miR-155 may regulate Bmal1 mRNA stability and translation. Nevertheless, researchers have not clearly determined whetheantagomir-155 upregulates BMAL1 expression and subsequently attenuates ICHinduced brain injury in rats.

Methods: After establishing an ICH rat model by injecting autologous blood, the time course of changes in levels of the BMAL1 protein after ICH was analyzed. Subsequently, this study was designed to investigate the potential role and mechanisms of BMAL1 in SBI following ICH using lentiviral overexpression and antagomir-155 treatments.

Results: BMAL1 protein levels were significantly decreased in the ICH group compared to the sham group. Genetic overexpression of BMAL1 alleviated oxidative stress, inflammation, brain edema, bloodbrain barrier injury, neuronal death, and neurological dysfunction induced by ICH. On the other hand, we observed that inhibiting miRNA-155 using antagomir-155 promoted the expression of BMAL1 and further activated the nuclear factor erythroid 2-related factor 2 (Nrf2) signaling pathway to attenuate brain injury after ICH.

Conclusions: These results reveal that BMAL1 serves as a neuroprotective agent in ICH and upregulation of BMAL1 attenuates ICH-induced SBI. Therefore, BMAL1 may be a promising therapeutic target for SBI following ICH.

Keywords: Intracerebral hemorrhage (ICH); secondary brain injury (SBI); brain and muscle Arnt-like protein 1 (BMAL1); microRNA-155

Submitted Apr 14, 2021. Accepted for publication Aug 31, 2021.

doi: $10.21037 / \mathrm{atm}-21-1863$

View this article at: https://dx.doi.org/10.21037/atm-21-1863 


\section{Introduction}

Intracerebral hemorrhage (ICH) is a severe cerebrovascular disease with high rates of morbidity and mortality (1). Currently, specific or effective treatment approaches for ICH are unavailable $(2,3)$. Previous studies have shown that secondary brain injury (SBI) following ICH is an important factor leading to neurological dysfunction $(1,4)$. The pathophysiological processes of SBI are related to many intricate mechanisms, including oxidative stress, hematoma toxicity, inflammation, cell apoptosis, blood-brain barrier (BBB) damage and mitochondrial dysfunction (5). Among these pathophysiological mechanisms, oxidative stress is an important sign of SBI following ICH and participates in various pathophysiological responses after $\operatorname{ICH}(5,6)$. Oxidative stress refers to the excessive production of free radicals, which mainly include reactive oxygen species (ROS). In addition, ICH-induced inflammation induces the production of many free radicals, which lead to cell damage $(1,7)$. Therefore, antioxidant therapy is regarded as a valuable and hopeful direction for the treatment of ICH.

Brain and muscle Arnt-like protein 1 (BMAL1) is the core element of the circadian clock, and it is the only clock protein whose deficiency leads to complete disturbance of all rhythms $(8,9)$. At present, the function of BMAL1 is not limited to core clock gene regulation, as it is also involved in regulating various pathological processes, such as oxidative stress, inflammation and BBB integrity $(10,11)$. Additionally, BMAL1 has been reported to activate nuclear factor erythroid 2-related factor 2 (Nrf2) signaling pathways and then alleviate inflammatory responses in lens epithelial cells (10), macrophages (12), and lungs (13). Therefore, BMAL1 is involved in regulating oxidative stress responses. However, researchers have not determined whether BMAL1 participates in the pathological processes of brain injury after ICH.

MicroRNAs are small noncoding endogenous RNA molecules that inhibit the activity of their target mRNAs (14). Among the various microRNAs, microRNA-155 (miR-155) is expressed in neurons (15) and participates in various pathological mechanisms, including oxidative stress and inflammation (16-18). Through a gene sequencing analysis, the GCAUUAA sequence was found to be present in the 3' untranslated region of BMAL1. In addition, a previous study showed that miR-155 inhibits BMAL1 by binding to this sequence in macrophages (19). Thus, miR-155 may regulate Bmal1 mRNA stability and translation. Moreover, a previous study suggested that strategies targeting miR-155 and its downstream signaling pathway exerted a beneficial effect on SBI induced by ICH (20). Nevertheless, researchers have not clearly determined whether antagomir-155 upregulates BMAL1 expression and subsequently attenuates ICH-induced brain injury in rats.

The aim of this study was to determine the role of BMAL1 and its related mechanisms in SBI following ICH. We hypothesized that upregulation of BMAL1 by inhibiting miR-155 attenuates ICH-induced SBI by regulating the Nrf2 signaling pathway. The findings of this study will contribute to the identification of potential therapeutic targets and innovative strategies for ICH.

We present the following article in accordance with the ARRIVE reporting checklist (available at https://dx.doi. org/10.21037/atm-21-1863).

\section{Methods}

\section{Materials}

Primary antibodies for western blot analysis and immunofluorescence staining, including Anti-BMAL1 antibody (MA5-25133) was obtained from Invitrogen (USA). Anti-NeuN (abl04224, abl7748), anti-Ibal (ab178846) and anti-GFAP (ab7260) antibodies were purchased from Abcam (USA). The anti- $\beta$-tubulin antibody (\#2146) was purchased from Cell Signaling Technology (USA).

Anti-Albumin (DF6396), anti-Nrf2 (AF0639) and antiHIF-1 $\alpha$ (AF1009) antibodies were purchased from Affinity Biosciences (China).

Secondary antibodies for western blot analysis, including goat anti-rabbit IgG-HRP (sc-2004) and goat anti-mouse IgG-HRP (sc-2005), were obtained from Santa Cruz Biotechnology (USA). Secondary antibodies for immunofluorescence staining, including the Alexa Fluor 555-conjugated donkey anti-mouse IgG antibody (A31570), Alexa Fluor 555-conjugated donkey anti-rabbit IgG antibody (A31572), Alexa Fluor 488-conjugated donkey anti-rabbit IgG antibody (A21206) and Alexa Fluor 488-conjugated donkey anti-mouse IgG antibody (A21202), were purchased from Invitrogen (USA).

Drugs and detection kits were used for this study, including the lentivirus for overexpressing BMAL1 (LVBMAL1, 43493-1) and controls (Ubi-MCS-3FLAGSV40-puromycin) were customized commercially by GeneChem Corporation (Shanghai, China). Antagomir-155 (miR30000165) and the negative control were purchased from RiboBio Corporation (Guangzhou, China). The M6 
primer (RmiRQ9003), miR-155 primer (RmiRQP8090), First-Strand cDNA synthesis kit (QP057), SYBR Green qPCR mix 2.0 (QP032), and All-in-one miRNATM qRTPCR and Detection Kit 2.0 (Q116) were purchased from GeneCopoeia (USA). TNF- $\alpha$ and IL- $1 \beta$ ELISA kits were purchased from Bio-Swamp (China). Reactive Oxygen Species Assay Kits were obtained from Beyotime Institute of Biotechnology (China). Cell death detection kit (TUNEL) was purchased from Roche (Germany).

\section{Animals}

The animal experiments described in this study were authorized by the Committee of the First Affiliated Hospital of Soochow University (No. 2020147) and conducted in compliance with the institutional guidelines for the care and use of animals. Adult male Sprague-Dawley (SD) rats (280$300 \mathrm{~g}$ ) were included in this study. All rats were housed at $40-60 \%$ humidity and $22-24{ }^{\circ} \mathrm{C}$. All rats were able to freely eat food and drink water. We tried to reduce animal numbers and suffering in this study.

\section{ICH model in rats}

Based on previous methodology (21), autologous whole blood was used to establish the ICH model in male SD rats. First, we administered $4 \%$ chloral hydrate through an intraperitoneal injection to anesthetize the rats. Then, the animals were immobilized in a stereotaxic apparatus. We selected the right basal ganglia as the injection site, which was $1.5 \mathrm{~mm}$ posterior, $3.5 \mathrm{~mm}$ lateral, and $5.5 \mathrm{~mm}$ below the horizontal plane of the bregma. Cardiac autogenous whole blood $(100 \mu \mathrm{L})$ was slowly and constantly injected into the right basal ganglia with a microliter syringe. Finally, the needle was left in place for another 5 minutes before the scalp was sutured. Sham group rats were injected with the same volume of the physiological saline solution. The graphical presentation of the establishment of the ICH model in rats is shown in Figure S1A.

\section{Intracerebroventricular lentivirus and antagomir-155 injection}

The intracerebroventricular injection of the lentivirus was conducted as described in previous studies $(22,23)$. The lentivirus overexpressing BMAL1 (LV-BMAL1) and control lentivirus were customized commercially by GeneChem Corporation (Shanghai, China). Five microliters of LV-
BMAL1 (containing $1 \times 10^{9}$ genome copies of Lenti. BMAL1 gene) or equivalent control lentiviruses were intracerebroventricularly injected into the rats. The period of lentiviral expression in the brain ranges from 72 hours to approximately 1 month after injection. In this study, we chose to inject the lentivirus one week before the ICH model was established.

The antagomir-155 injection was performed as described in previous studies $(24,25)$. A scrambled miRNA served as the negative control (NC). Antagomir-155 and the negative control were purchased from RiboBio (Guangzhou, China). Antagomir-155 or antagomir-NC was dissolved in sterile saline to the appropriate final concentration $(0.2 \mathrm{nmol} / \mu \mathrm{L}$, total $10 \mu \mathrm{L}$ ) and then intracerebroventricularly injected into each rat 1 day before establishing the ICH model.

According to a previous report (26), intracerebroventricular injections were performed $0.9 \mathrm{~mm}$ caudal to the posterior and $1.9 \mathrm{~mm}$ right lateral to the bregma. A leveled microliter syringe $(10 \mu \mathrm{L})$ attached to a stereotaxic manipulator was lowered $3.5 \mathrm{~mm}$ into the brain. To reduce counterflow, the needle remained in place for 5 minutes before suturing the scalp to reduce counterflow.

\section{Experimental groups and study design}

The experimental groups were randomly allocated. Using a random-number table, rats were distributed by a technician who was blinded to all groups. Before the ICH model was established, all rats were randomly divided into sham and ICH groups. After the ICH model was constructed, these ICH rats were randomly allocated into different subgroups according to the random number table by the same technician. Details are presented below.

\section{Experiment 1: time course analysis of BMAL1 protein levels after ICH}

Forty-eight rats (54 rats were used, but only 48 rats survived after the surgery) were randomly assigned to the sham group and ICH group. Then, the ICH group was divided into seven subgroups for analysis at different time points, including 3 h, 6 h, 12 h, 24 h, 48 h, 72 h and 7 days after ICH. Brain tissues were collected from all experimental rats at the same time to avoid the effect of the collection time on BMAL1 levels in the samples. Then, centering on the bleeding point of brain tissues from the right hemisphere, $3 \mathrm{~mm}$ sections of specimens preceding the bleeding point were selected as the samples for western blots, while $4 \mathrm{~mm}$ 
sections of specimens behind the bleeding point were used for immunofluorescence analyses (Figure S1B).

\section{Experiment 2: the role of BMAL1 in brain injury after ICH in rats}

One hundred seven rats were used. Ninety-six rats that survived after the surgery were included in this experiment and then randomly assigned into four groups: sham group, $\mathrm{ICH}$ group, ICH + Vector group, and ICH + BMAL1 overexpression (Over-BMAL1) group. ICH + Vector group: ICH rats were treated with the empty vector. ICH + OverBMAL1 group: ICH rats were treated with the BMAL1 overexpression lentiviral vector. ICH + Vector group: ICH rats treated with the empty vector. ICH + Over-BMAL1 group: ICH rats treated with BMAL1 overexpression lentiviral vector. The time point of ICH group was $24 \mathrm{~h}$ after ICH in this experiment. Twenty-four rats in each group were further divided randomly into three subgroups for different experimental purposes. First, at $24 \mathrm{~h}$ after $\mathrm{ICH}$, we collected blood samples from six rats in each group for ELISAs of interleukin-1 $\beta$ (IL-1 $\beta$ ) and tumor necrosis factor- $\alpha(\mathrm{TNF}-\alpha)$. Brain tissues were immediately collected from these 6 rats in each group at the same time. Then, these samples were used for western blot analysis, Fluoro-Jade B (FJB) and Nissl staining, and terminal deoxynucleotidyl transferase-mediated dUTP nick end labeling (TUNEL) staining. Second, another 6 rats were used to observe brain edema. Finally, the last 12 rats in each group were subjected to neurobehavioral tests (Figure S1C).

\section{Experiment 3: the effects and related mechanisms of BMAL1 upregulation upon inbibiting miR-155 during brain injury in rats}

Ninety-six rats (96 of 108 rats that survived in this experiment) were randomly divided into four groups: sham group, ICH group, ICH + antagomir-NC (negative control) group, and ICH + antagomir-155 group. In experiment 3 , the time point of ICH group was also $24 \mathrm{~h}$ after ICH. Twenty-four rats in each group were randomly allocated to three subgroups. For the first test, at $24 \mathrm{~h}$ after ICH, blood samples were collected from six rats in each group for ELISAs of IL- $1 \beta$ and TNF- $\alpha$ levels. Brain tissues from these 6 rats in each group were immediately collected at the same time. These specimens were subjected to western blot analysis and Nissl staining. For the second subgroup, another 6 rats in each group were used to detect the brain water content. For the last subgroup, the remaining 12 rats in each group were subjected to neurobehavioral tests (Figure S1D).

\section{Western blot analysis}

Based on previous studies, brain samples around the hematoma were collected from SD rats. Then, icecold RIPA lysis buffer (Beyotime, China) was used to mechanically triturate and lyse these samples before they were centrifuged for $10 \mathrm{~min}\left(4^{\circ} \mathrm{C}, 12,000 \mathrm{~g}\right)$. Afterward, the supernatant liquid was collected and enhanced BCA Protein Assay Kits (Beyotime) were used to measure the protein concentrations. Forty micrograms of protein extracts from each group were loaded into $8 \%$ or $10 \%$ SDS polyacrylamide gels, separated and electrically transferred to nitrocellulose membranes (Millipore Corporation, Billerica, MA, USA). Subsequently, the membrane was blocked with $5 \%$ bovine serum Albumin (BSA, BioSharp, China) for $1 \mathrm{~h}$ at room temperature. Afterward, the membrane was incubated with primary antibodies at $4{ }^{\circ} \mathrm{C}$ overnight. In this study, we used primary antibodies, including BMAL1 (1:500) (Thermo Fisher Scientific, Invitrogen, USA), Nrf2 (1:500), HIF-1 $\alpha$ (1:500), Albumin (1:500) (Affinity, China) and $\beta$-tubulin $(1: 2,000)$ (Cell Signaling Technology, USA), which served as the loading control. Then, we incubated the membrane with an HRP-conjugated secondary antibody for $1 \mathrm{~h}$ at $23-25^{\circ} \mathrm{C}$. Finally, we detected band signals using an enhanced chemiluminescence kit (Beyotime, China) and analyzed the relative levels of proteins with ImageJ software (NIH, Bethesda, MD, USA). A technician blinded to the experimental groups performed the analysis.

\section{RT-PCR}

The protocol for the RT PCR assay of miRNA expression has been elaborated in previous studies (27). The protocol generally consists of two steps: the reverse transcription of RNA into complementary DNA (cDNA) and the actual RT-PCR step. Specific kits (QP116, GeneCopoeia, USA) and qPCR primers (RmiRQP0890, RmiRQP9003, GeneCopoeia, USA) were used for RT-PCR. U6 served as the loading control.

\section{Immunofluorescent analysis}

The brain tissues around the hematoma were fixed with $4 \%$ paraformaldehyde, embedded in paraffin, cut into 
$4 \mu \mathrm{m}$ sections, and dewaxed before staining. After antigen retrieval, we incubated the sections from each group with primary antibodies against BMAL1 (1:100), NeuN (1:500), Iba1 (1:500) and GFAP (1:500) for $12 \mathrm{~h}$ at $4{ }^{\circ} \mathrm{C}$ and homologous secondary antibodies for $1 \mathrm{~h}$ at $25^{\circ} \mathrm{C}$. Subsequently, we performed DAPI (Southern Biotech, USA) staining of nuclei, and then coverslips were used to protect the sections. Ultimately, six random sections of brain tissues around the hematoma from each group were observed under a fluorescence microscope (Nikon, Japan), and the relative intensities were evaluated using ImageJ software. The experiments, images and quantification of the samples were performed by blinded observers using the same microscope configurations to eliminate bias due to background normalization.

\section{Nissl staining}

Coronal sections around the hematoma were first dewaxed and rehydrated, and then these sections were stained with a toluidine blue solution for $40 \mathrm{~min}$ at $55^{\circ} \mathrm{C}$. Afterward, the sections were dehydrated stepwise in a series of ethanol solutions for $3 \mathrm{~min}$, including one incubation each with $70 \%, 80 \%$, and $95 \%$ ethanol and two incubations with $100 \%$ ethanol. Then, all sections were immersed in two solutions of $100 \%$ dimethylbenzene for ten minutes, and coverslipped with neutral balsam. Finally, we counted the numbers of surviving neurons per $400 \times$ field of the cortex and hippocampal region.

\section{FFB staining}

In this study, we applied FJB staining to evaluate neuronal degradation. After the sections around the hematoma had been deparaffinized and dehydrated, the slides were immersed in a $0.06 \%$ potassium permanganate solution (Sigma-Aldrich, USA) for $15 \mathrm{~min}$. Subsequently, these slides were cleared with deionized water, immersed in FJB working solution for $30 \mathrm{~min}$, and dried in an incubator. Finally, the sections were washed with xylene for $2 \mathrm{~min}$, and neutral balsam was applied to cover the sections. We observed the FJB-positive cells using fluorescence microscopy and a technician who was blinded to the experimental conditions counted the cells.

\section{TUNEL staining}

TUNEL staining was applied to detect cell death in tissue sections. As described previously, the slices of rat brain sections were immersed in $0.1 \%$ Triton $\mathrm{X}-100$ for $5 \mathrm{~min}$ and rinsed with PBS three times. Afterward, we incubated these sections with TUNEL staining reagents at $37{ }^{\circ} \mathrm{C}$ for $1 \mathrm{~h}$. Finally, we used a fluorescence microscope (Nikon, Japan) to observe and analyze the TUNEL-positive cells in the brain tissues around the hematoma.

\section{ELISA}

Twenty-four hours after establishing the ICH model, we collected blood samples from each rat by puncturing the heart under anesthesia. The blood samples were centrifuged at $1,000 \mathrm{~g}$ for $5 \mathrm{~min}$ at $4{ }^{\circ} \mathrm{C}$. Then, we collected the supernatants. These supernatants were used to detect the levels of TNF- $\alpha$ and IL- $1 \beta$ using specific ELISA kits (Bio-Swamp, China) according to the manufacturer's instructions.

\section{Reactive oxygen species (ROS) detection assay}

ROS levels were detected using the Reactive Oxygen Species Assay Kit (Beyotime Institute of Biotechnology, Shanghai, China). The collected brain tissue was homogenized and then centrifuged $(12,000 \mathrm{~g})$ for $10 \mathrm{~min}$ at $4^{\circ} \mathrm{C}$, and the supernatants were collected for the ROS assay. We used the oxidant-sensitive probe 2,7-dichlorofluorescein diacetate (DCF-DA) to detect ROS levels according to the manufacturer's instructions. A fluorometric microplate reader (FilterMax F5, Molecular Devices, Sunnyvale, CA, USA) was applied to evaluate the fluorescence intensity of each sample. ROS levels are reported as fluorescence intensity/mass of total protein $(\mathrm{mg})$, and the data from each group were normalized to the sham group.

\section{BBB injury and brain edema}

Albumin extravasation is regarded as an index of $\mathrm{BBB}$ permeability. In general, due to the integrity of the BBB, the level of the Albumin protein in brain tissues is often very low. However, once the BBB is damaged, Albumin levels in brain tissues are significantly increased. Therefore, levels of the Albumin protein were tested to assess the degree of BBB injury. Levels of the Albumin protein in brain tissues from rats in each group were detected using western blot analysis.

According to our previous study (28), at $24 \mathrm{~h}$ after $\mathrm{ICH}$, the brain tissues of rats were collected at the same 
time. Then, the brain tissue of each rat was divided into five parts, including the ipsilateral cortex, ipsilateral basal ganglia, contralateral basal ganglia, contralateral cortex and cerebellum. The wet weight of each tissue was measured with an electronic analytical balance. Then, each sample was dried in a thermostatic dryer at $100{ }^{\circ} \mathrm{C}$ for $72 \mathrm{~h}$, and the dry weight was recorded. In this study, we adopted the following formula to calculate brain water content: (wet weight - dry weight)/wet weight $\times 100 \%$.

\section{Neurobehavioral evaluation}

In this study, we calculated modified Garcia scores to evaluate short-term neurobehavioral function. At $24 \mathrm{~h}$ after ICH, 12 rats in each group were subjected to an analysis of modified Garcia scores, which included seven subtests, as described by Chen et al. (29). Each score ranged from 0 to 3 points, and when the rat did not have neurological dysfunction, the maximum score was 21 . This test was carried out by a researcher who was blinded to the experimental conditions.

The adhesive-removal test and rotarod test were performed at baseline (pre-ICH) and 1, 7, 14, and 20 days after ICH, as previously described (23), to further evaluate long-term neurologic functions, Moreover, the Morris water maze was conducted at 21 to 26 days after ICH to assess the learning and memory functions of rats in each group (30). After rats found the submerged platform, a computer tracking system (Noldus Ethovision, Tacoma, WA) was used to record their swimming path, escape latency, and swimming distance at 21 and 25 days after ICH. Then, after the platform was removed, we carried out the probe trial at 26 days after ICH to observe the time spent in the probe quadrant.

\section{Statistical analysis}

GraphPad Prism 8 software (GraphPad Software, San Diego, USA) was used for statistical analyses. All data are described as the means \pm standard errors of the means (SEM), and Kolmogorov-Smirnov tests were used to test for normality of datasets in each group. One-way analyses of variance (ANOVAs) were applied to analyze the measurement data with normal distributions, whereas Mann-Whitney $U$ tests were performed to analyze nonparametric data. All statistical tests and $\mathrm{P}$ values were two-sided, and $\mathrm{P}<0.05$ was considered statistically significant.

\section{Results}

\section{Protein levels of BMAL1 decreased in brain tissue of rats after ICH}

We performed the western blot analysis of brain tissues and double immunofluorescence staining of brain sections to detect levels of the BMAL1 protein in rat brain tissues at different time points following ICH. The results of the western blot analysis showed that ICH induced a significant decrease in the level of the BMAL1 protein compared to the sham group (Figure $1 \mathrm{~A}, 1 \mathrm{~B}$ ). After the ICH model was established, BMAL1 levels were reduced as early as $12 \mathrm{~h}$, and the lowest level was observed at $24 \mathrm{~h}$ after ICH, which subsequently increased sharply after $48 \mathrm{~h}$ and then increased gradually. Therefore, the time point of $24 \mathrm{~h}$ after ICH was chosen for the subsequent experiments. Moreover, double immunofluorescence staining was applied to distinguish changes in the cell type specificity of BMAL1 expression at $24 \mathrm{~h}$ after ICH. Compared with the sham group, a remarkably decreased number of BMAL1-positive neurons was observed in rat brain tissues at $24 \mathrm{~h}$ after ICH, while no significant changes in microglia and astrocytes between the sham and ICH groups, which were shown in Figure $1 \mathrm{C}-1 \mathrm{~F}$.

\section{Effects of BMAL1 overexpression on oxidative stress, BBB injury, brain edema, inflammation and neurobehaviors after ICH}

We established lentivirus-mediated overexpression of BMAL1 (Over-BMAL1) in ICH rats to determine the role of BMAL1 in brain injury after ICH. The controls for Over-BMAL1 were a scrambled lentivirus composed of an empty vector (Vector). Using western blotting, we confirmed that transfection with the recombinant lentivirus (Over-BMAL1) significantly increased BMAL1 expression levels in ICH rats (Figure 2A,2B). The ROS level served as an indicator of oxidative stress. Obviously increased ROS levels were detected in the ICH group compared to the sham group. However, ROS levels were significantly lower in the ICH + Over BMAL1 group than in the ICH + Vector group (Figure 2C). Based on these results, increased BMAL1 expression alleviated oxidative stress in ICH rats. We used western blot analysis to measure the levels of Albumin in brain tissues following ICH and to confirm the role of BMAL1 in BBB injury. The results revealed an obvious increase in the Albumin level in the ICH group compared to the sham group. Furthermore, BMAL1 overexpression significantly alleviated ICH-induced Albumin extravasation 


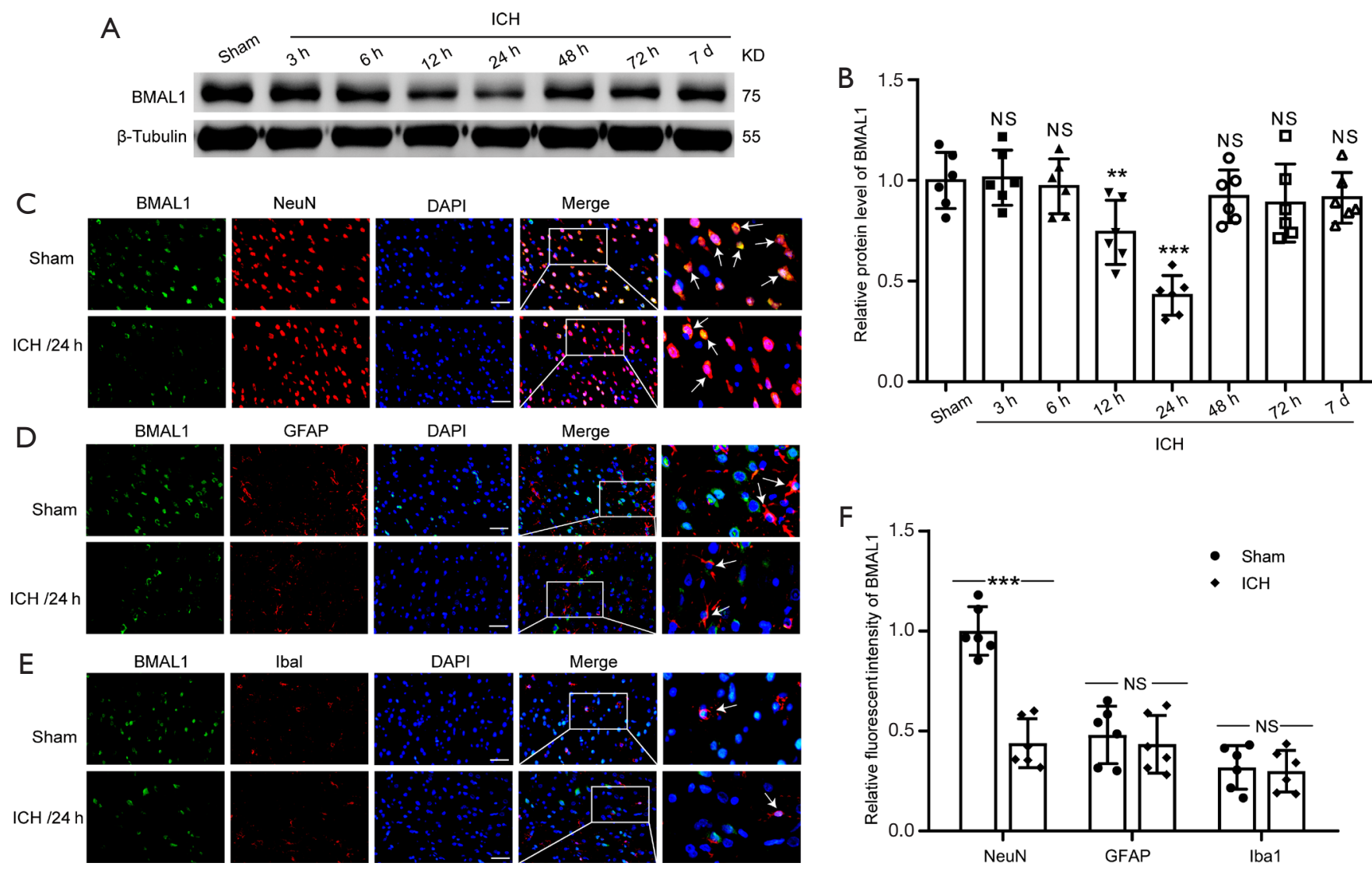

Figure 1 Protein levels of BMAL1 in brain tissues after ICH in rats. (A) Western blot analysis of the relative BMAL1 expression levels in the rat brain tissues at various time points. (B) Quantification of the BMAL1 protein levels at various time points. (C) Double immunofluorescence staining was performed in brain sections using antibodies against BMAL1 (green) and a neuronal marker (red). Nuclei were fluorescently labeled with DAPI (blue). Arrow heads indicate BMAL1/NeuN-positive cells. (D) Double immunofluorescence staining was performed with antibodies against BMAL1 (green) and an astrocyte marker (GFAP, red) in brain sections. Nuclei were fluorescently labeled with DAPI (blue). Arrow heads indicate BMAL1/GFAP-positive cells. (E) Double immunofluorescence staining was performed with antibodies against BMAL1 (green) and a microglial marker (Iba1, red) in brain sections. Nuclei were fluorescently labeled with DAPI (blue). Arrow heads indicate BMAL1/Iba1-positive cells. Representative images from the sham and ICH (24 h) groups are shown. (F) Relative fluorescence intensity of BMAL1 with NeuN (neurons), GFAP (astrocytes) and Iba1 (microglia) in the brain at $24 \mathrm{~h}$ after ICH. Scale bar $=50 \mu \mathrm{m}$. All data are presented as the means $\pm \mathrm{SEM},{ }^{* *} \mathrm{P}<0.01$ vs. Sham group; ${ }^{* * *} \mathrm{P}<0.001$ vs. Sham group; NS, no significant difference $v s$. Sham group. n=6. ICH, Intracerebral hemorrhage; DAPI, 4',6-diamidino-2-phenylindole; SEM, standard error of mean.

(Figure 2A,2D). Additionally, the water content of the $\mathrm{ICH}$ group was significantly higher than that of the sham group. However, the brain water content of the ICH + OverBMAL1 group was significantly lower than that of the $\mathrm{ICH}$ + Vector group (Figure 2E). These data emphasized the protective effects of BMAL1 on ICH-induced brain edema. In addition, the serum levels of IL- $1 \beta$ and TNF- $\alpha$ in rats from each group were detected to further explore the effects of BMAL1 on inflammation. We found significantly higher levels of IL- $1 \beta$ and TNF- $\alpha$ in the ICH group than those in the sham group. In contrast, serum IL-1 $\beta$ and TNF- $\alpha$ levels were remarkably reduced in the ICH + Over-BMAL1 group (Figure $2 F-2 G$ ), which indicated an anti-inflammatory role of BMAL1 in ICH.

We performed a modified Garcia score test, rotarod test and Morris water maze to determine ICH-induced neurological deficits and whether BMAL1 overexpression exerted an effect on the behavior of rats. At $24 \mathrm{~h}$ after ICH, modified Garcia scores of rats in each group were evaluated. Compared to the sham group, the modified Garcia scores 
A
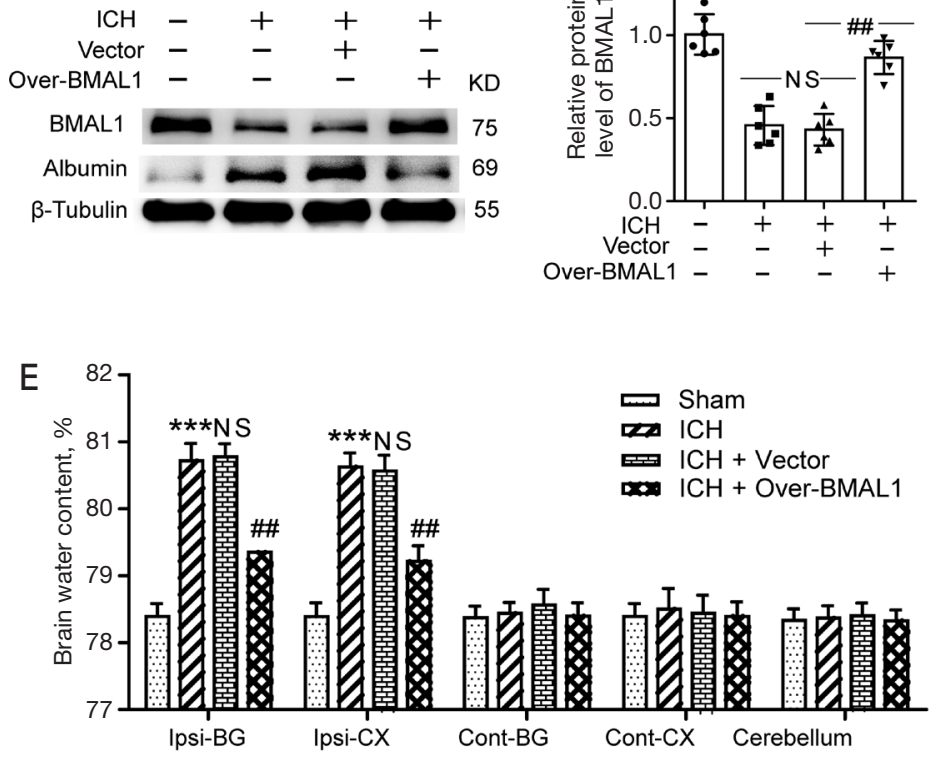

B

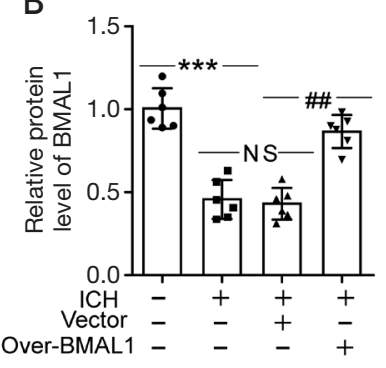

C

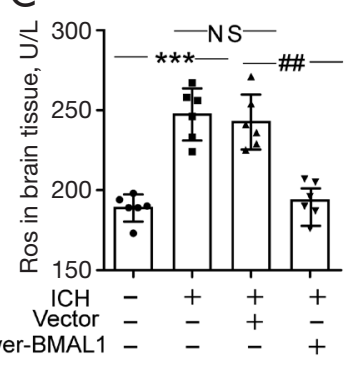

Over-BMAL1 - $-\quad+\quad+$

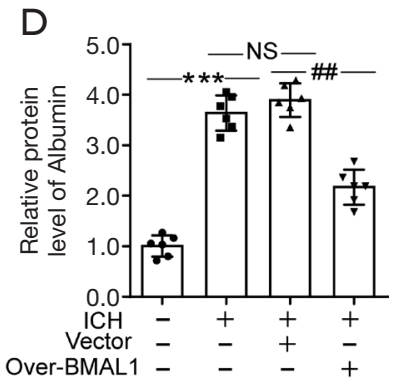

$\mathrm{F}$
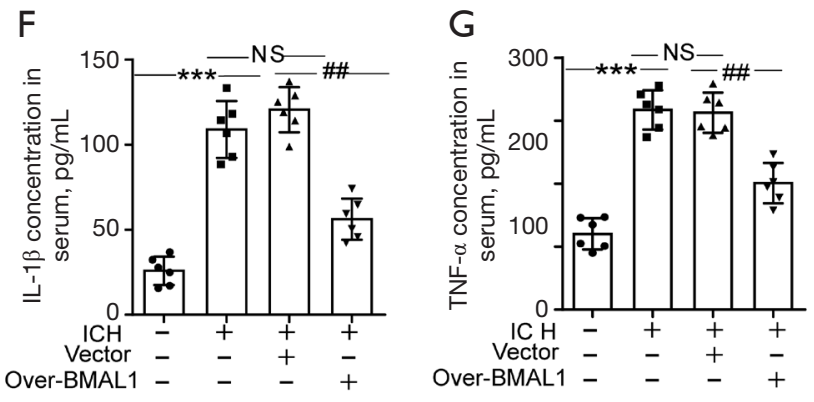
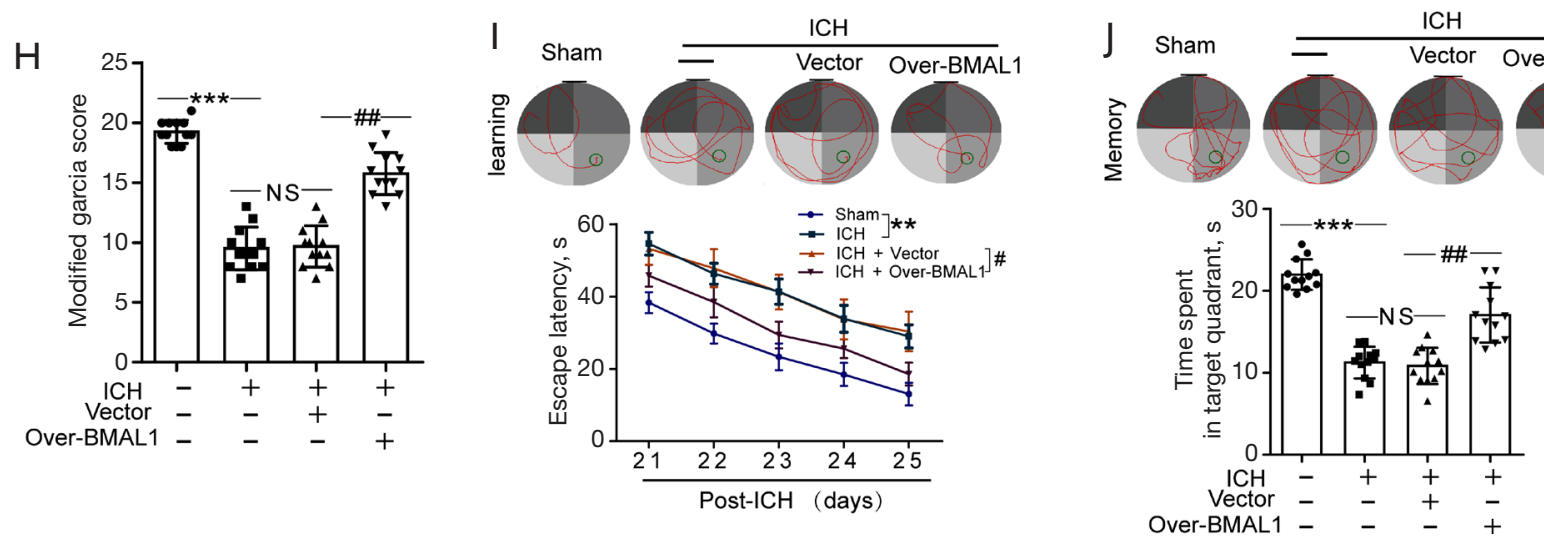

Figure 2 Effects of BMAL1 overexpression on oxidative stress, BBB injury, brain edema, inflammation and neurobehaviors after ICH. (A) Western blot analysis of levels of the BMAL1 and Albumin proteins (index of BBB injury) in the sham, ICH, ICH + Vector and ICH + Over-BMAL1 groups at $24 \mathrm{~h}$ after ICH. (B) Quantification of the relative BMAL1 protein levels in the four groups. (C) ROS levels in the brain tissues from rats in the four groups. (D) Quantification of the relative Albumin protein levels in the four groups. (E) The effects of BMAL1 overexpression on the brain water content of rats in the four groups. (F) Concentrations of IL-1 $\beta$ in the serum of the sham ICH, $\mathrm{ICH}+$ Vector and ICH+Over-BMAL1 groups at $24 \mathrm{~h}$ after ICH. (G) Concentrations of TNF- $\alpha$ in the serum of the four groups listed above. $(\mathrm{H})$ The scores on the modified Garcia test in the sham, ICH, ICH + Vector and ICH + Over-BMAL1 groups at $24 \mathrm{~h}$ after ICH. (I) Representative images illustrate swimming trajectories at $25 \mathrm{~d}$ (learning) and escape latency in the Morris water maze test at 21 to $25 \mathrm{~d}$ after ICH in the sham, ICH, ICH + Vector and ICH + Over BMAL1 rats. (J) Representative images illustrate swimming trajectories at $26 \mathrm{~d}$ (memory) and time spent in the platform quadrant in the Morris water maze test at $26 \mathrm{~d}$ after ICH in the groups listed above. ${ }^{* *} \mathrm{P}<0.01 v s$. Sham group; ${ }^{* * *} \mathrm{P}<0.001$ vs. Sham group; ${ }^{*} \mathrm{P}<0.05$ vs. ICH + Vector group; ${ }^{{ }^{* \prime}} \mathrm{P}<0.01$ vs. ICH + Vector group. NS, no significant difference vs. ICH group. In (A-G), n=6; In (H-J), n=10. BBB, blood brain barrier; ICH, intracerebral hemorrhage; ROS, reactive oxygen species. 
of rats in the ICH group were significantly decreased at $24 \mathrm{~h}$ after ICH. However, BMAL1 overexpression obviously increased the modified Garcia scores after ICH (Figure 2H). A Morris water maze was carried out from 21 to 26 days after ICH to evaluate cognition and further confirm whether upregulation of BMAL1 by overexpression attenuated brain injury in rats after ICH. More serious cognitive dysfunction was observed in ICH rats. However, the ICH + Over-BMAL1 group spent less time searching for the platform, revealing that BMAL1 overexpression significantly improved spatial learning (Figure 2I). Twentysix days after sham or ICH surgery, the platform was removed, and memory tests were carried out. Rats in the $\mathrm{ICH}+$ Over-BMAL1 group spent more time in the target platform quadrant than those in the ICH + Vector group (Figure 2f). Based on these results, BMAL1 overexpression improved the cognitive function of ICH rats.

\section{Effects of BMAL1 overexpression on neuronal injury and degeneration induced by $\mathrm{ICH}$}

Nissl staining (Figure $3 A$ ) was performed to assess neuronal injury in the brain tissues of ICH rats. TUNEL staining (Figure 3B) and FJB staining (Figure 3C) were applied to evaluate the role of BMAL1 in neuronal death and degeneration in rat brain tissues after ICH. Nissl staining showed significantly reduced numbers of surviving neurons in the cortex and hippocampus regions after $\mathrm{ICH}$. However, the numbers of surviving neurons were significantly increased in the ICH + Over-BMAL1 group (Figure $3 A, 3 D, 3 E$ ). In addition, overexpression of BMAL1 obviously reduced the number of TUNEL-positive neurons (Figure 3B,3F). Similarly, BMAL1 overexpression significantly decreased the number of FJB-positive cells (Figure 3C,3G). Thus, increased levels of the BMAL1 protein mitigated neuronal cell death and degeneration following ICH.

\section{BMAL1 expression increased in ICH rats after antagomir-155 treatment}

TargetScan was used to identify the downstream genes of miR-155. The prediction revealed that BMAL1 might be a potential target gene of miR-155. Figure $4 A$ illustrates the binding site for BMAL1 in miR-155. First, we performed RT-PCR to investigate miR-155 expression in rat brain tissues after ICH. The levels of miR-155 were increased after $\mathrm{ICH}$, and the increase was established at $3 \mathrm{~h}$ after
ICH and persisted at a high level (Figure 4B). Then, we used western blotting analysis to detect miRNA-155mediated changes in BMAL1 expression following the administration of antagomir-155. The results of the western blot analysis showed that administration of antagomir-155 markedly increased the BMAL1 protein level in ICH rats (Figure 4C,4D).

\section{Effects of antagomir-155 on oxidative stress, inflammation, neuronal injury and brain edema after ICH}

At $24 \mathrm{~h}$ after ICH, compared to the ICH + antagomir-NC group, ROS levels were significantly decreased in the ICH + antagomir-155 group (Figure 5A). The results suggested that increased BMAL1 expression via antagomir-155 alleviated oxidative stress in ICH rats. Serum levels of IL- $1 \beta$ and TNF- $\alpha$ in rats from each group were detected to further explore the effects of BMAL1 on inflammation. Serum IL-1 $\beta$ and TNF- $\alpha$ levels were remarkably reduced in the ICH + antagomir-155 group (Figure 5B, 5C). Nissl staining showed significantly increased numbers of surviving neurons in the cortex and hippocampus in the ICH + antagomir-155 group compared to the ICH + antagomir$\mathrm{NC}$ group (Figure $5 D-5 F$ ). In addition, the brain water content of the $\mathrm{ICH}+$ antagomir-155 group was significantly lower than that of the ICH + antagomir-NC group (Figure $5 G$ ). These data emphasized the protective effects of BMAL1 on ICH-induced brain edema.

\section{Effects of antagomir-155 on neurological function after ICH in rats}

We performed the modified Garcia score test, rotarod test and Morris water maze to determine the effects of antagomir-155 on the neurological function of rats after ICH. At $24 \mathrm{~h}$ after ICH, modified Garcia scores were evaluated for the rats in each group. Compared to the ICH + antagomir-NC group, the modified Garcia scores of rats in the $\mathrm{ICH}+$ antagomir-155 group were significantly increased at $24 \mathrm{~h}$ after ICH (Figure 6A). Rotarod tests were performed to detect the locomotor activity of rats at days 7,14 , and 20 after ICH and to further examine the effect of BMAL1 overexpression on functional recovery after ICH. The results of rotarod tests showed that rats in the $\mathrm{ICH}+$ antagomir-155 group manifested significantly longer latency times than those in the ICH + antagomir-NC group (Figure 6B). Moreover, the adhesive removal test was used to evaluate sensorimotor function. Rats in the ICH 

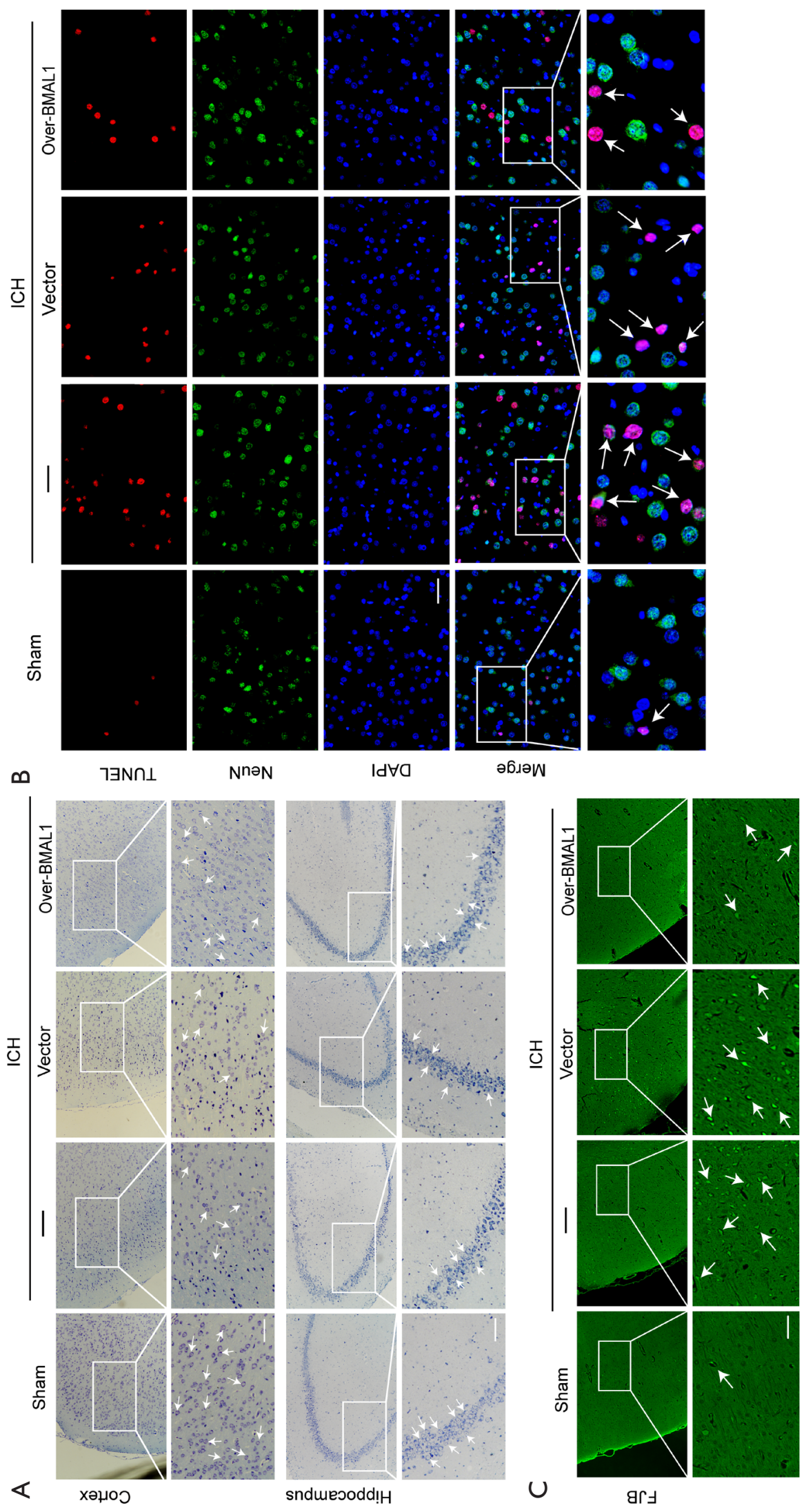

(C) Annals of Translational Medicine. All rights reserved. 

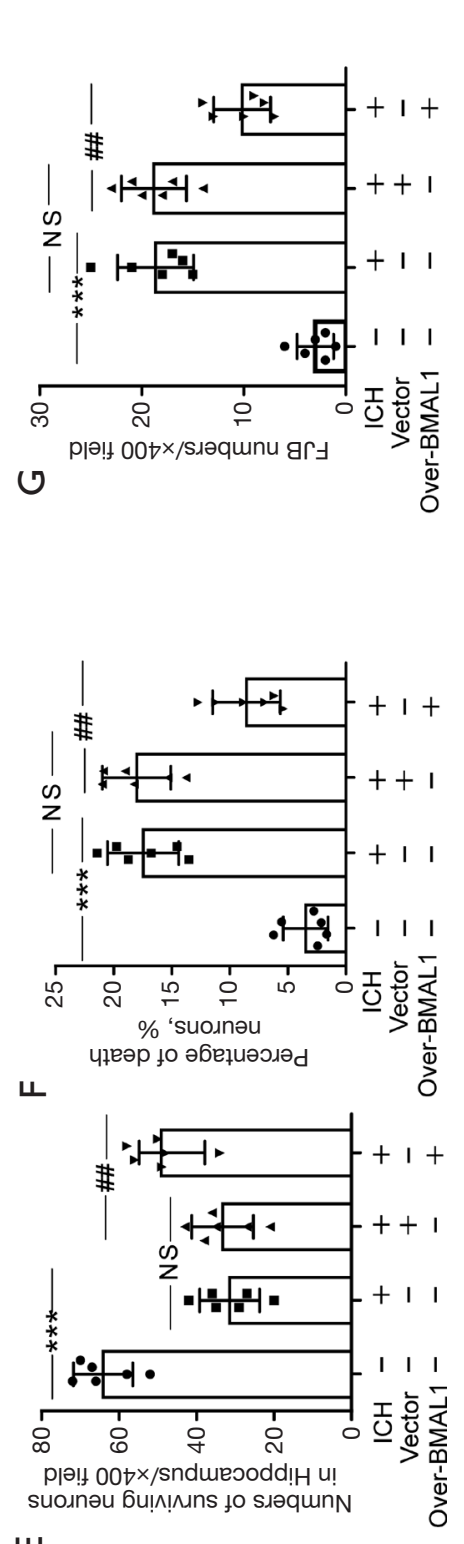

ш

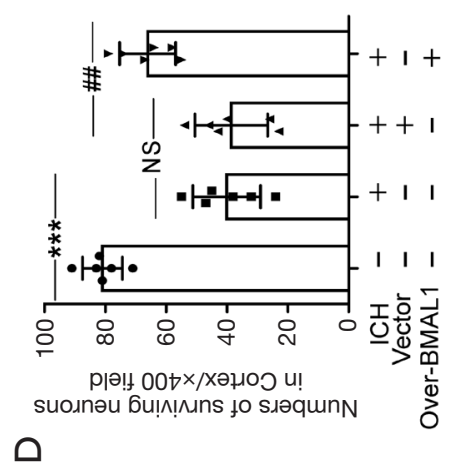

口 group manifested sensorimotor dysfunction, as revealed by significantly shorter latencies until contact and removal of the tape from the forepaws. However, ICH + antagomir-155 rats showed the opposite effect (Figure 6C,6D). The Morris water maze was carried out to evaluate cognition from 21 to 26 days after ICH. The results of the Morris water maze showed that rats in the $\mathrm{ICH}+$ antagomir-155 group spent less time searching for the platform (Figure 6E). Twentysix days after sham or ICH surgery, the platform was removed, and memory tests were carried out. Rats in the $\mathrm{ICH}+$ antagomir-155 group spent more time in the target platform quadrant than those in the $\mathrm{ICH}+$ antagomir-NC group (Figure 6F). These results revealed that increased BMAL1 expression mediated by antagomir-155 improved the cognitive function of ICH rats, including spatial learning and memory function.

\section{Effects of increased BMAL1 on the Nrf2 signaling pathway after ICH}

Protein levels of the BMAL1, Nrf2 and HIF-1 $\alpha$ in the brain tissue of $\mathrm{ICH}$ rats were detected using western blotting after antagomir-155 treatment to determine whether increased BMAL1 expression activated the Nrf2 signaling pathway to attenuate brain injury after ICH in rats. The level of the BMAL1 protein was significantly increased after antagomir-155 treatment (Figure 7A,7B). Compared to $\mathrm{ICH}+\mathrm{NC}$ group, the $\mathrm{Nrf} 2$ protein level was remarkably increased in the ICH + antagomir-155 group (Figure 7A,7C). Additionally, the protein level of HIF-1 $\alpha$ in $\mathrm{ICH}+$ antagomir-155 group was lower than that in $\mathrm{ICH}+$ antagomir-NC group (Figure $7 A, 7 D$ ).

Taken together, these results indicated that the upregulation of BMAL1 by antagomir-155 activated the Nrf2 signaling pathway and then attenuated SBI induced by $\mathrm{ICH}$, including oxidative stress, inflammation, and neuronal death (Figure 7E).

\section{Discussion}

This study mainly explored the role of BMAL1 in SBI after ICH by establishing a rat ICH model through the injection of autologous blood in vivo. Lentivirus-mediated overexpression of BMAL1 was performed to determine the effects of BMAL1 on ICH-induced SBI. Furthermore, the protective effect of BMAL1 on brain injury after ICH was observed after treatment with antagomir-155, and the Nrf2 signaling pathway was also investigated. As shown in 

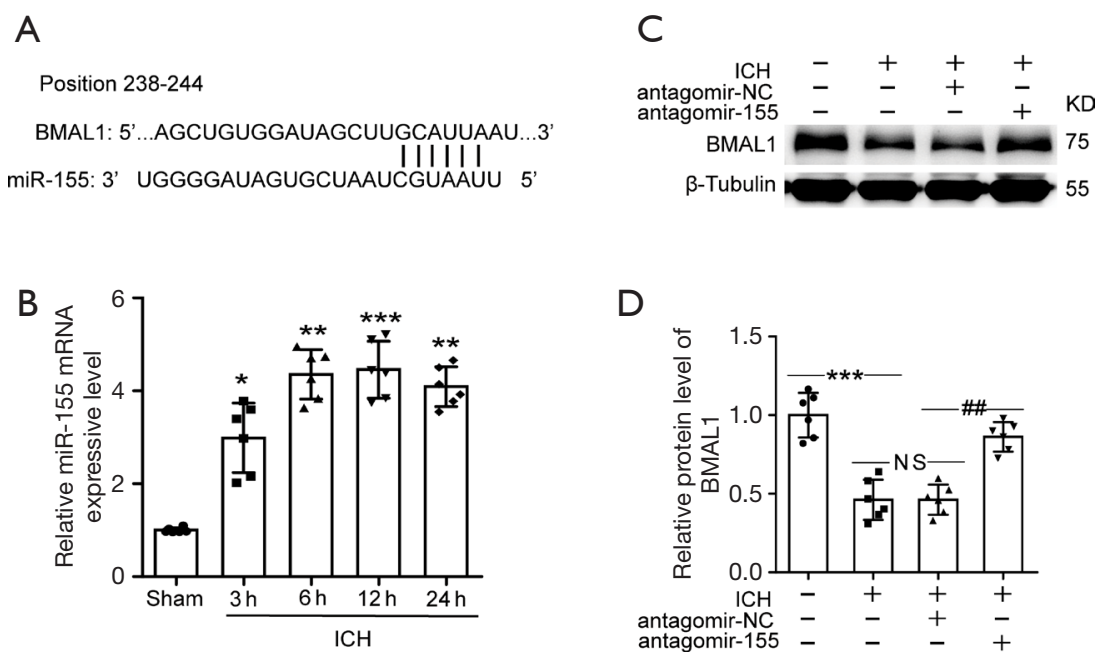

Figure 4 The miR-155 expression level and level of the BMAL1 protein by inhibiting miR-155 after ICH. (A) The binding site of BMAL1 in the miR-155 3'UTR. (B) The levels of miR-155 were increased after ICH, and the increase was established at $3 \mathrm{~h}$ after ICH and remained at a high level. (C) Western blot analysis of the BMAL1 protein levels in the sham, ICH, ICH + antagomir- $\mathrm{NC}$ and ICH + antagomir-155 groups. (D) Quantification of the relative BMAL1 protein levels in the groups listed above. All data are presented as the means \pm SEM, ${ }^{*} \mathrm{P}<0.05$ vs. Sham group; ${ }^{* *} \mathrm{P}<0.01$ vs. Sham group; ${ }^{* *} \mathrm{P}<0.001$ vs. Sham group; ${ }^{\# \#} \mathrm{P}<0.01$ vs. ICH + antagomir-NC group; NS, no significant difference $v s$. ICH group; n=6. ICH, intracerebral hemorrhage; NC, negative control.

the present study, the upregulation of BMAL1 activated the Nrf2 signaling pathway and further attenuated ICHinduced SBI.

$\mathrm{ICH}$ is a common type of stroke that often results in the disability and death of patients. Compared with ischemic stroke, the global burden of death and economic burden associated with the treatment of ICH are obviously higher $(31,32)$. ICH mainly occurs in the basal ganglia region and causes proximal or distal white matter damage, including axon damage, demyelination, and synaptic changes, ultimately leading to severe motor dysfunction and a poor prognosis of patients with ICH (33). I In addition, cognitive dysfunction is one of the main complications after ICH. Although the regions of hippocampus and cortex are not in situ hemorrhagic foci, ICH can lead to secondary nerve injury, including neuronal death and apoptosis in the hippocampus and cortex $(5,34)$. In the present study, ICH rats exhibited cognitive decline, and the hippocampus and cortex presented neuronal death and apoptosis-related changes, consistent with previous studies $(35,36)$. The mechanisms of brain injury after ICH include not only the initial mechanical injury induced by the hematoma mass effect, but also subsequent damage called SBI (1). The pathophysiological mechanisms of SBI after ICH mainly include oxidative stress, inflammation, cytotoxicity and cell death (including necrosis and apoptosis). Among them, oxidative stress and inflammatory responses often occur together and interact with each other. Inflammatory responses in the brain might occur soon after ICH and peak several days later $(37,38)$. During the inflammatory response after $\mathrm{ICH}$, activation of neutrophils results in the destruction of the respiratory chain and the release of abundant ROS (39). When oxygen free radicals are overproduced or clearance is weakened, cell damage or death will occur. Moreover, these pathophysiological responses can cause multiple pathological events in the brain after ICH, such as BBB damage and brain edema, which eventually lead to more comprehensive brain injury (34). Therefore, most studies have focused on effective treatments for SBI induced by ICH.

BMAL1 is a core element of the biological clock. However, BMAL1 not only plays a role in the regulation of clock genes but can also regulates the transcription of downstream genes in addition to clock genes (40). Previous studies have established a link between abnormalities in BMAL1 and several pathological states, such as myocardial ischemia and Parkinson's disease $(41,42)$. Moreover, research has reported that a targeted deficiency of Bmal1 leads to severe cardiac dysfunction and a subsequent increase in mortality $(9,43)$. Hu et al. also indicated 

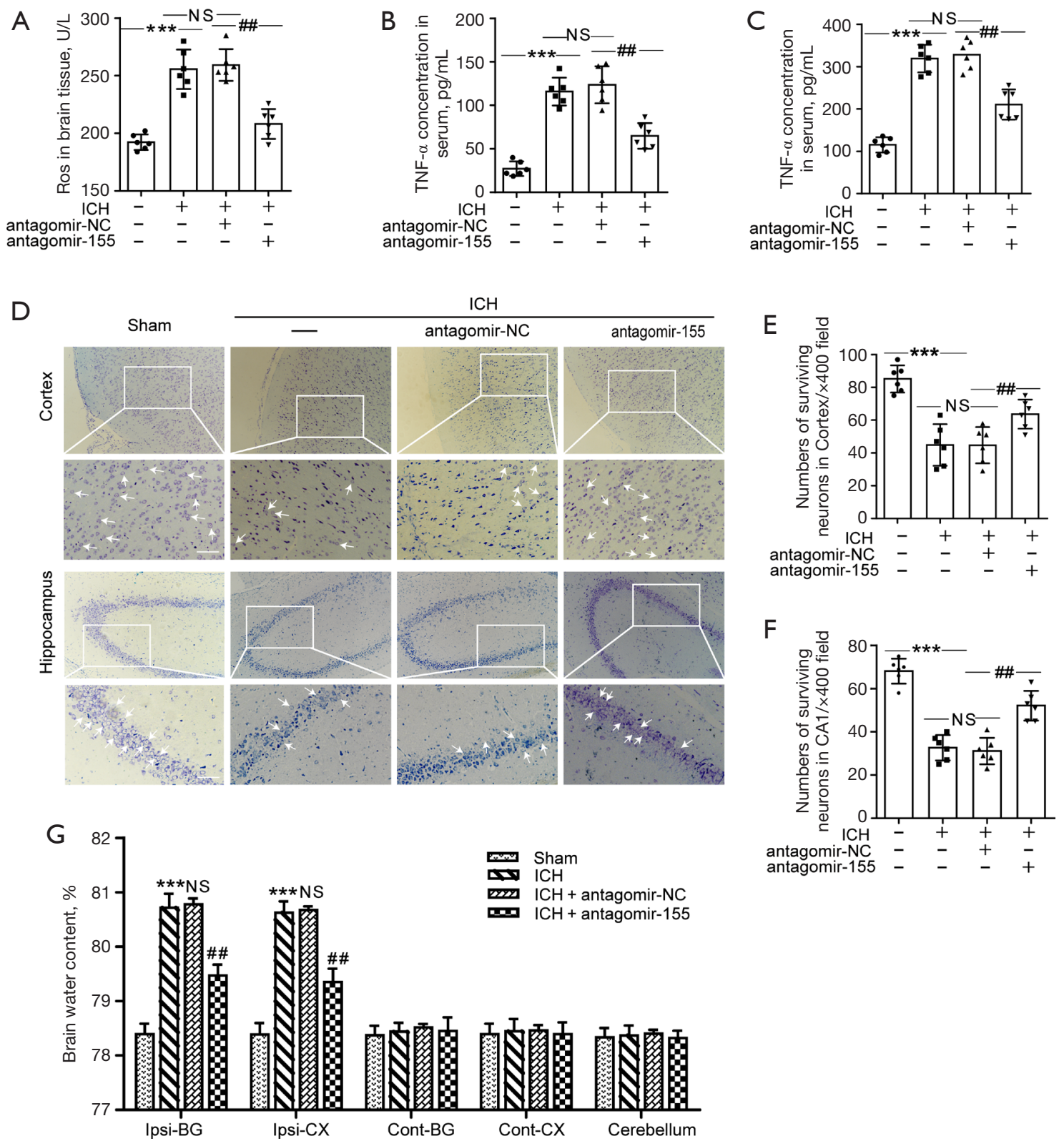

Figure 5 Effects of antagomir-155 on oxidative stress, inflammation, neuronal injury and brain edema after ICH. (A) ROS levels in the brain tissues of rats in the sham, ICH, ICH + antagomir-NC and ICH + antagomir-155 groups at $24 \mathrm{~h}$ after ICH. (B) Concentrations of IL-1 $\beta$ in the serum of the sham, ICH, ICH + antagomir-NC and ICH + antagomir-155 groups at 24 h after ICH. (C) Concentrations of TNF- $\alpha$ in the serum of the four groups listed above. (D) Nissl staining was used to assess neuronal death in the sham, ICH, ICH + antagomir- $\mathrm{NC}$ and $\mathrm{ICH}+$ antagomir-155 groups at $24 \mathrm{~h}$ after ICH. Arrows indicate surviving neurons. Scale bar =50 $\mu \mathrm{m}$. (E,F) Quantitative analysis of surviving neurons in the cortex and hippocampus of each group. The numbers of surviving neurons were counted per 400x field. (G) The effects of increased BMAL1 expression on the brain water content of rats in the four groups. All data are presented as the means \pm SEM, ${ }^{* * *} \mathrm{P}<0.001$ vs. Sham group; ${ }^{\# \#} \mathrm{P}<0.01$ vs. ICH + antagomir-NC group; NS, no significant difference vs. ICH group; $\mathrm{n}=6 . \mathrm{ICH}$, intracerebral hemorrhage; ROS, reactive oxygen species; NC, negative control; SEM, standard error of mean. 

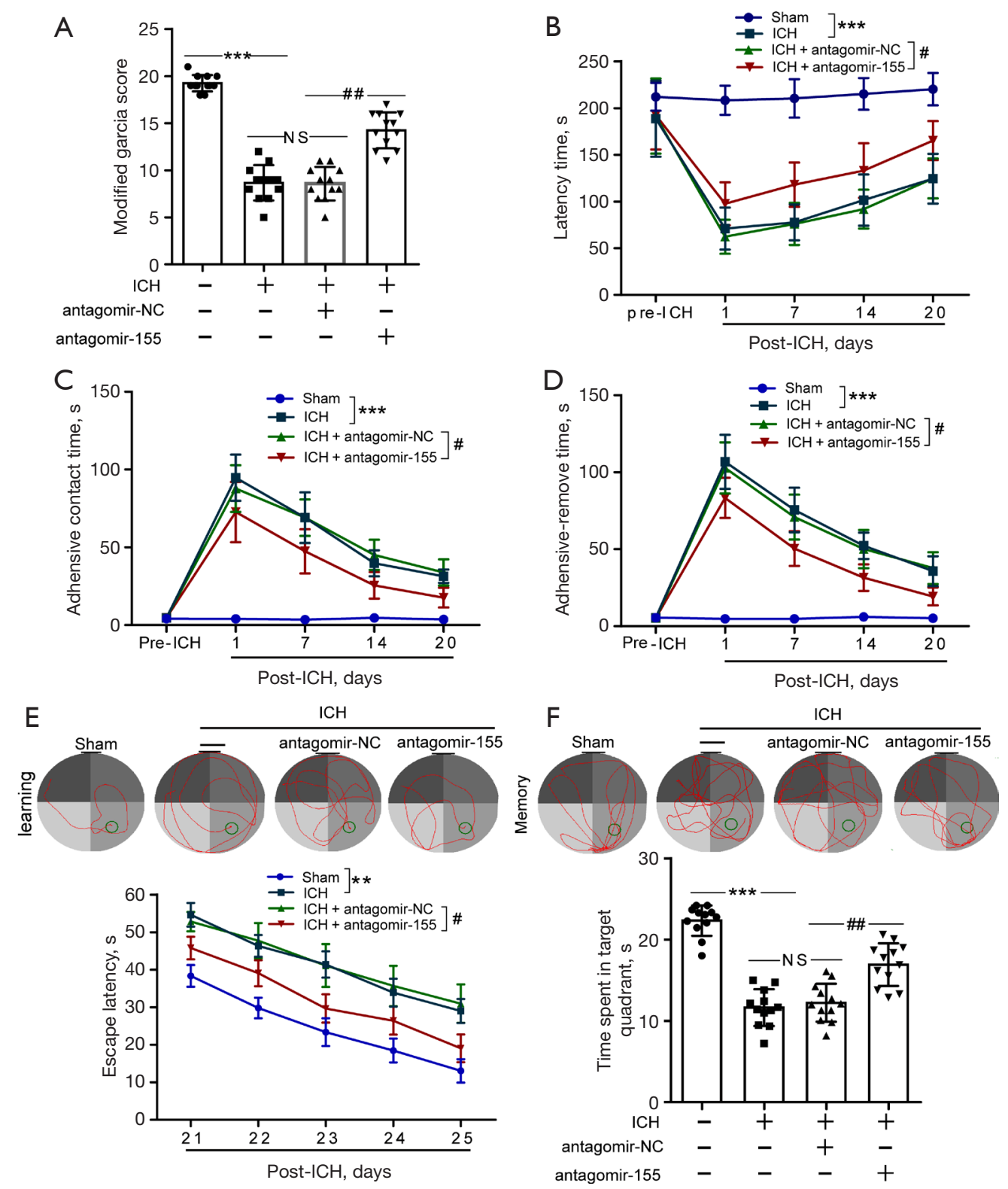

Figure 6 Effects of antagomir-155 on neurological function after ICH in rats. (A) The scores on the modified Garcia test in the sham, $\mathrm{ICH}, \mathrm{ICH}+$ antagomir-NC and ICH + antagomir-155 groups at $24 \mathrm{~h}$ after ICH. (B) The latency to fall in the rotarod test before (3 d of pretraining before $\mathrm{ICH}$ ) and up to $20 \mathrm{~d}$ after ICH in the various groups. (C) Time to feel the adhesive tapes in the adhesive removal test before and up to $20 \mathrm{~d}$ after ICH. (D) Time to remove the adhesive tapes in the adhesive removal test before and up to $20 \mathrm{~d}$ after ICH. (E) Representative images illustrate swimming trajectories at $25 \mathrm{~d}$ (learning) and escape latency in the Morris water maze test at 21 to $25 \mathrm{~d}$ after $\mathrm{ICH}$ in the sham, ICH, ICH + antagomir-NC and ICH + antagomir-155 groups. (F) Representative images illustrate swimming trajectories at $26 \mathrm{~d}$ (memory) and time spent in the platform quadrant in the Morris water maze test at $26 \mathrm{~d}$ after ICH in the groups listed above. All data are presented as the means \pm SEM. ${ }^{* * *} \mathrm{P}<0.001$ vs. Sham group; ${ }^{* *} \mathrm{P}<0.01$ vs. Sham group; ${ }^{\#} \mathrm{P}<0.05$ vs. ICH + antagomir-NC group; ${ }^{\#} \mathrm{P}<0.01$ vs. ICH + antagomir-NC group; NS, no significant difference vs. ICH group; In (A-D), n=12, In (E,F), n=10. ICH, intracerebral hemorrhage; NC, negative control. 


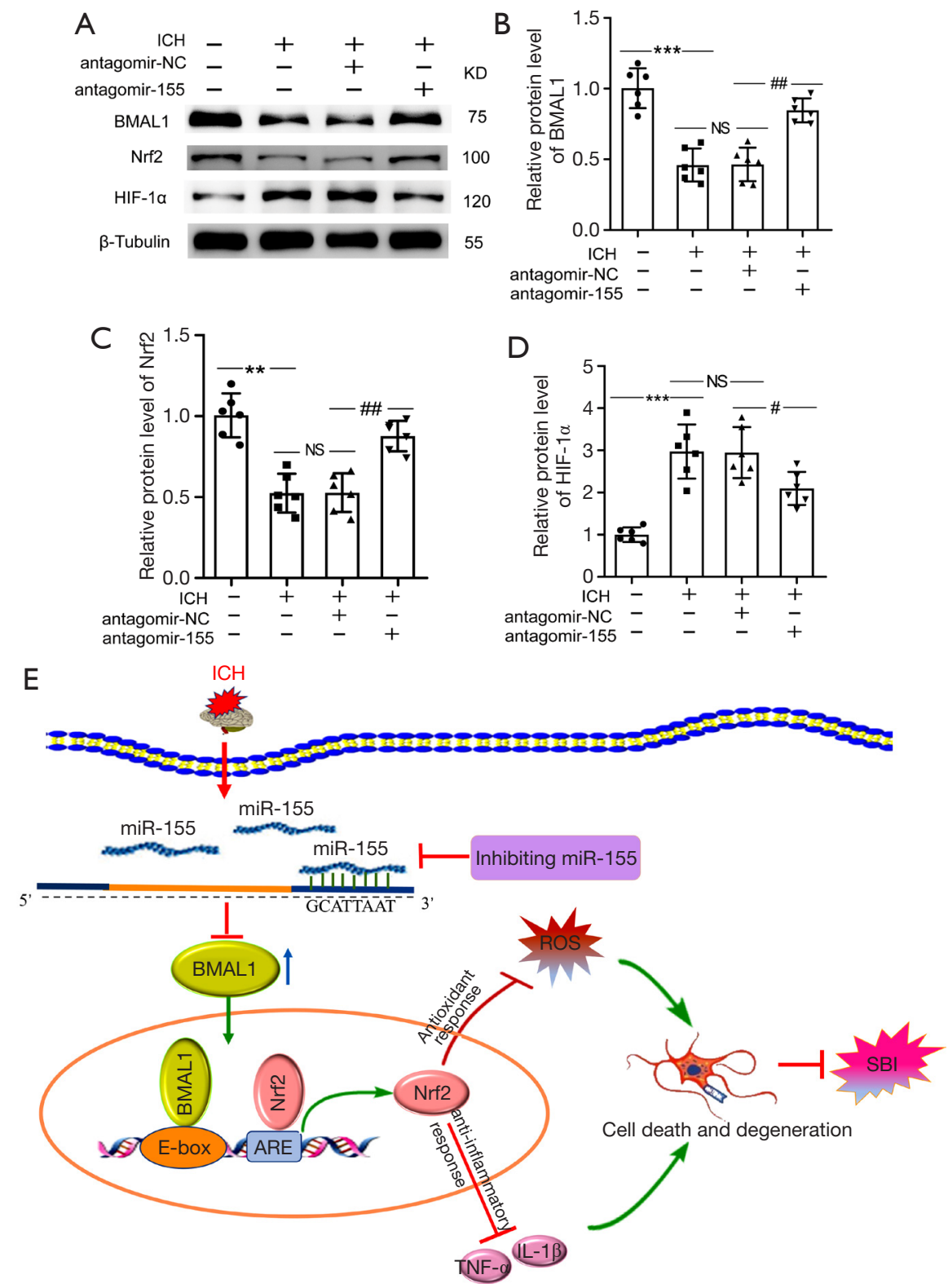

Figure 7 Effects of increased BMAL1 on the Nrf2 signaling pathway after ICH. (A) Western blot analysis of the relative levels of the BMAL1, Nrf2 and HIF-1 $\alpha$ proteins in the sham, ICH, ICH + antagomir-NC and ICH + antagomir-155 groups at $24 \mathrm{~h}$ after ICH; (B) Western blot analysis of the relative levels of the BMAL1 protein in the groups listed above; (C) Western blot analysis of the relative levels of the Nrf2 protein in the groups listed above; (D) Western blot analysis of the relative levels of the HIF-1 $\alpha$ protein in the groups listed above; (E) Schematic representation of the role and related mechanism of BMAL1 in SBI after ICH. The BMAL1 protein level decreased in the brain tissue of rats after ICH. After antagomir-155 treatment, the BMAL1 protein was upregulated, and then the Nrf2 signaling pathway was activated to attenuate SBI induced by $\mathrm{ICH}$, including oxidative stress, inflammation, and neuronal death. All data are presented as the mean \pm SEM. ${ }^{* *} \mathrm{P}<0.001$ vs. Sham group; ${ }^{* *} \mathrm{P}<0.01$ vs. Sham group; ${ }^{\# \#} \mathrm{P}<0.01$ vs. $\mathrm{ICH}+$ antagomir-NC group; ${ }^{\#} \mathrm{P}<0.05$ vs. ICH + antagomirNC group; NS, no significant difference $v s$. ICH group; n=6. ICH, intracerebral hemorrhage; NC, negative control; SEM, standard error of mean. 
that the expression level of Bmall exerted an effect on cardiomyocyte function including ROS production (41). In addition, downregulation of BMAL1 expression affects colon carcinoma cell oxidative stress, apoptosis and fate decisions (44), and aggravates various pathological mechanisms, such as oxidative stress and inflammatory reactions. Previous epidemiological studies showed that the disturbance of circadian rhythm was involved in the occurrence and progression of ICH $(45,46)$. Nevertheless, to date, no studies have determined the role of BMAL1 in $\mathrm{ICH}$, and researchers have not clearly determined whether an abnormality in BMAL1 expression occurs after ICH. In this study, we explored the role of BMAL1 in an ICH rat model with autologous blood injection for the first time. Levels of the BMAL1 protein were obviously reduced, and the lowest level was detected at $24 \mathrm{~h}$ after ICH. This finding suggested that decreased BMAL1 expression may be involved in the pathological processes of SBI after ICH, including oxidative stress and inflammatory responses. Additionally, we further investigated whether increased BMAL1 protein levels attenuated brain injury after ICH. Our results showed that BMAL1 overexpression significantly reduced brain injury, including increasing the number of surviving neurons, mitigating brain edema and $\mathrm{BBB}$ injury, improving neurological function, and alleviating inflammation and oxidative stress. Therefore, these findings reveal that increased BMAL1 attenuates SBI and pathological responses after ICH.

MicroRNAs directly regulate genes through posttranscriptional mechanisms by inhibiting mRNA translation or inducing their cleavage and degradation (47). According to previous studies, several miRNAs directly regulate Bmal1 mRNA stability and translation, including miR-155, miR-211, and miR-142-3p $(19,48,49)$. Among these microRNAs, miR-155 is identified as an important regulatory component of circadian function and is related to various pathophysiological processes involved in oxidative stress and neuroinflammation, including controlling the production of inflammatory cytokines $(19,50)$. Moreover, Zhang et al. reported that there was a striking increase in miR-155 levels after ICH, and antagomir-155 attenuated neural injury after ICH by reducing inflammation and oxidative stress (20). According to previous reports, miR-155 is a critical posttranscriptional repressor of Bmal1 in macrophages and endothelial cells $(19,51)$. Based on these findings, we hypothesized that increased levels of miR-155 following ICH inhibit Bmall gene expression and then exacerbate ICH-induced brain injury. We verified this hypothesis by further determining that BMAL1 protein levels were upregulated by antagomir-155 to attenuate neuronal injury following ICH. The antagomir-155 treatment significantly increased the BMAL1 protein level after ICH and further attenuated oxidative stress, inflammation, and neuronal dysfunction in ICH rats.

In addition to BMAL1, other target genes of miR-155 have been identified, such as cytokine signaling 1 (SOCS1), which is a critical regulator of inflammation (52). Moreover, miR-155 is also involved in regulating stroke development by promoting the expression of TNF- $\alpha$ and IL- $1 \beta$ (53). SIRT1 is also a direct target of miR-155, and a miR-155 inhibitor upregulates SIRT1 expression (54). Therefore, the upregulation of SIRT1 may exert protective effects on ICHinduced mitochondrial injury (55). These results reveal that inhibition of miR-155 may represent a new therapeutic strategy and direction for the treatment of $\mathrm{ICH}$.

Oxidative stress is one of the main causes of brain injury after ICH. It participates in various important pathophysiological processes after ICH (7). Oxidative stress causes BBB injury, irreversible damage to neurovascular structures, serious brain edema and brain cell death $(5,34)$. The primary characteristic of oxidative stress is excess ROS production. ROS also induce the acute expression of proinflammatory cytokines, including TNF- $\alpha$ and IL-1 $\beta$ (56). Recently, emerging evidence has indicated that BMAL1 is vital for regulating oxidative responses and the inflammatory response $(8,10,12,42)$. Nrf2 plays a vital role in regulating the expression of major antioxidant genes and mediating antioxidant and anti-inflammatory responses. Currently, accumulating evidence has shown that Bmal1 directly regulates the Nrf2 signaling pathway by binding to its response element E-Box $(8,12,13)$. Because oxidative stress and inflammatory responses are important pathological mechanisms of ICH-induced SBI, our study further determined whether the upregulation of BMAL1 using antagomir-155 activated the Nrf2 signaling pathway to attenuate brain injury induced by ICH. The Nrf2 protein level was increased and the Nrf2 signaling pathway was activated upon the upregulation of BMAL1, including reduced ROS and proinflammatory cytokine production, consistent with previous studies $(12,57)$. Consistent with previous studies, these findings also revealed that the cellular abundance of BMAL1 is essential for the regulation of the Nrf2 signaling pathway $(10,58)$.

Our results showed an increase in the BMAL1 protein level at $24 \mathrm{~h}$ after ICH due to overexpression and antagomir-155 treatments. Moreover, increased BMAL1 
expression activated the Nrf2 signaling pathway and obviously attenuated brain injury and neurological deficits after ICH. These findings suggest that BMAL1 exerts its potential neuroprotective effect by suppressing oxidative stress and inflammatory responses (Figure $7 D$ ). BMAL1 is the core element of the circadian clock, and thus abnormal BMAL1 expression leads to a disrupted circadian rhythm. At present, an increasing number of studies have shown that circadian rhythm disruption affects the occurrence, progression and clinical prognosis of ICH (59-61). Additionally, circadian biology may interact with multiple pathophysiological processes in stroke, including nerve cell responses, cytokines and chemokines, endothelial or hemostasis mechanisms, immune responses, temperature regulation, $\mathrm{BBB}$, drug delivery, or metabolism $(62,63)$. The circadian rhythm disruption and abnormal expression of related rhythm molecules are novel molecular mechanisms involved in brain injury after ICH. Therefore, this study provides potential directions for the further development of new therapies for ICH.

The current study has some limitations. First, in this study, we used only healthy adult male SD rats to establish the ICH model, but most patients are older and female in the clinic. Therefore, animals of different sexes and ages should be tested in subsequent studies. Second, we did not further confirm whether the downregulation of BMAL1 would aggravate brain injury induced by ICH. In addition, the mechanisms by which the upregulation of BMAL1 via inhibition of miR-155 attenuated brain injury after ICH were not investigated in vitro. Third, we chose $24 \mathrm{~h}$ after ICH as the treatment point for the subsequent investigations based on the results of the first experiment, but further exploration is needed to determine whether other times are more suitable. Finally, one RNAbinding protein corresponds to multiple target mRNAs, and one mRNA is also regulated by multiple RNA-binding proteins and miRNAs. Therefore, further investigations are necessary to identify the roles of BMAL1 and miR-155 in brain injury after ICH.

\section{Conclusions}

In summary, this study is the first to determine that ICH decreased BMAL1 expression in rats. Overexpression of BMAL1 reduced oxidative stress, inflammation, neuronal death and neurobehavioral impairments. Furthermore, upregulation of BMAL1 using antagomir-155 attenuated ICH-induced SBI, possibly by regulating the Nrf2 signaling pathway. These results reveal that BMAL1 serves as a neuroprotective agent in brain injury after ICH. BMAL1 may be a potential therapeutic target for SBI following ICH.

\section{Acknowledgments}

Funding: The National Natural Science Foundation of China, grant number 82071297 . The science and technology planning project of Suzhou, grant number SYS2019102. Suzhou New District medical and health science and technology planning project, grant number 2017Q007. Suzhou 9th batch of 2019 science and technology development plan project, grant number 2016YFC0105904SFY.

\section{Footnote}

Reporting Checklist: The authors have completed the ARRIVE reporting checklist. Available at https://dx.doi. org/10.21037/atm-21-1863

Data Sharing Statement: Available at https://dx.doi. org/10.21037/atm-21-1863

Conflicts of Interest: All authors have completed the ICMJE uniform disclosure form (available at https://dx.doi. org/10.21037/atm-21-1863). The authors have no conflicts of interest to declare.

Ethical Statement: The authors are accountable for all aspects of the work in ensuring that questions related to the accuracy or integrity of any part of the work are appropriately investigated and resolved. The animal experiments of this study were authorized by the Committee of the First Affiliated Hospital of Soochow University (No. 2020147), in compliance with the institutional guidelines for the care and use of animals.

Open Access Statement: This is an Open Access article distributed in accordance with the Creative Commons Attribution-NonCommercial-NoDerivs 4.0 International License (CC BY-NC-ND 4.0), which permits the noncommercial replication and distribution of the article with the strict proviso that no changes or edits are made and the original work is properly cited (including links to both the formal publication through the relevant DOI and the license). See: https://creativecommons.org/licenses/by-nc-nd/4.0/. 


\section{References}

1. Keep RF, Hua Y, Xi G. Intracerebral haemorrhage: mechanisms of injury and therapeutic targets. Lancet Neurol 2012;11:720-31.

2. Ren H, Han R, Chen X, et al. Potential therapeutic targets for intracerebral hemorrhage-associated inflammation: An update. J Cereb Blood Flow Metab 2020;40:1752-68.

3. Cordonnier C, Demchuk A, Ziai W, et al. Intracerebral haemorrhage: current approaches to acute management. Lancet 2018;392:1257-68.

4. Urday S, Kimberly WT, Beslow LA, et al. Targeting secondary injury in intracerebral haemorrhage-perihaematomal oedema. Nat Rev Neurol 2015;11:111-22.

5. Aronowski J, Zhao X. Molecular pathophysiology of cerebral hemorrhage: secondary brain injury. Stroke 2011;42:1781-6.

6. Shao Z, Tu S, Shao A. Pathophysiological Mechanisms and Potential Therapeutic Targets in Intracerebral Hemorrhage. Front Pharmacol 2019;10:1079.

7. Yao Z, Bai Q, Wang G. Mechanisms of Oxidative Stress and Therapeutic Targets following Intracerebral Hemorrhage. Oxid Med Cell Longev 2021;2021:8815441.

8. Kondratov RV, Vykhovanets O, Kondratova AA, et al. Antioxidant $\mathrm{N}$-acetyl-L-cysteine ameliorates symptoms of premature aging associated with the deficiency of the circadian protein BMAL1. Aging (Albany NY) 2009;1:979-87.

9. Ray S, Valekunja UK, Stangherlin A, et al. Circadian rhythms in the absence of the clock gene Bmal1. Science 2020;367:800-6.

10. Chhunchha B, Kubo E, Singh DP. Clock Protein Bmal1 and Nrf2 Cooperatively Control Aging or Oxidative Response and Redox Homeostasis by Regulating Rhythmic Expression of Prdx6. Cells 2020;9:1861.

11. Nakazato R, Kawabe K, Yamada D, et al. Disruption of Bmal1 Impairs Blood-Brain Barrier Integrity via Pericyte Dysfunction. J Neurosci 2017;37:10052-62.

12. Early JO, Menon D, Wyse CA, et al. Circadian clock protein BMAL1 regulates IL- $1 \beta$ in macrophages via NRF2. Proc Natl Acad Sci U S A 2018;115:E8460-8.

13. Pekovic-Vaughan V, Gibbs J, Yoshitane H, et al. The circadian clock regulates rhythmic activation of the NRF2/ glutathione-mediated antioxidant defense pathway to modulate pulmonary fibrosis. Genes Dev 2014;28:548-60.

14. $\mathrm{Pu}$ M, Chen J, Tao Z, et al. Regulatory network of miRNA on its target: coordination between transcriptional and post-transcriptional regulation of gene expression. Cell
Mol Life Sci 2019;76:441-51.

15. Herai RR, Stefanacci L, Hrvoj-Mihic B, et al. Micro RNA detection in long-term fixed tissue of cortical glutamatergic pyramidal neurons after targeted lasercapture neuroanatomical microdissection. J Neurosci Methods 2014;235:76-82.

16. Miao F, Wang R, Cui G, et al. Engagement of MicroRNA-155 in Exaggerated Oxidative Stress Signal and TRPA1 in the Dorsal Horn of the Spinal Cord and Neuropathic Pain During Chemotherapeutic Oxaliplatin. Neurotox Res 2019;36:712-23.

17. Guo J, Liu HB, Sun C, et al. MicroRNA-155 Promotes Myocardial Infarction-Induced Apoptosis by Targeting RNA-Binding Protein QKI. Oxid Med Cell Longev 2019;2019:4579806.

18. Pasca S, Jurj A, Petrushev B, et al. MicroRNA-155 Implication in M1 Polarization and the Impact in Inflammatory Diseases. Front Immunol 2020;11:625.

19. Curtis AM, Fagundes CT, Yang G, et al. Circadian control of innate immunity in macrophages by miR-155 targeting Bmal1. Proc Natl Acad Sci U S A 2015;112:7231-6.

20. Zhang W, Wang L, Wang R, et al. A blockade of microRNA-155 signal pathway has a beneficial effect on neural injury after intracerebral haemorrhage via reduction in neuroinflammation and oxidative stress. Arch Physiol Biochem 2020. [Epub ahead of print]. doi: 10.1080/13813455.2020.1764047.

21. Shen F, Xu X, Yu Z, et al. Rbfox-1 contributes to CaMKII $\alpha$ expression and intracerebral hemorrhage-induced secondary brain injury via blocking micro-RNA-124. J Cereb Blood Flow Metab 2021;41:530-45.

22. Wang S, Li D, Huang C, et al. Overexpression of adiponectin alleviates intracerebral hemorrhage-induced brain injury in rats via suppression of oxidative stress. Neurosci Lett 2018;681:110-6.

23. Xue T, Sun Q, Zhang Y, et al. Phosphorylation at S548 as a Functional Switch of Sterile Alpha and TIR MotifContaining 1 in Cerebral Ischemia/Reperfusion Injury in Rats. Mol Neurobiol 2021;58:453-69.

24. Pang XM, Liu JL, Li JP, et al. Fastigial nucleus stimulation regulates neuroprotection via induction of a novel microRNA, rno-miR-676-1, in middle cerebral artery occlusion rats. J Neurochem 2015;133:926-34.

25. Krützfeldt J, Rajewsky N, Braich R, et al. Silencing of microRNAs in vivo with 'antagomirs'. Nature 2005;438:685-9.

26. Yang B, Wang S, Yu S, et al. C1q/tumor necrosis factor-related protein 3 inhibits oxidative stress during 
intracerebral hemorrhage via PKA signaling. Brain Res 2017;1657:176-84.

27. Chen G, Wang D, Zhao X, et al. miR-155-5p modulates malignant behaviors of hepatocellular carcinoma by directly targeting CTHRC1 and indirectly regulating GSK-3 $\beta$-involved Wnt/ $\beta$-catenin signaling. Cancer Cell Int 2017;17:118.

28. Zhang P, Wang T, Zhang D, et al. Exploration of MST1Mediated Secondary Brain Injury Induced by Intracerebral Hemorrhage in Rats via Hippo Signaling Pathway. Transl Stroke Res 2019;10:729-43.

29. Chen S, Zhao L, Sherchan P, et al. Activation of melanocortin receptor 4 with $\mathrm{RO} 27-3225$ attenuates neuroinflammation through AMPK/JNK/p38 MAPK pathway after intracerebral hemorrhage in mice. J Neuroinflammation 2018;15:106.

30. Wu J, He J, Tian X, et al. Activation of the Hedgehog Pathway Promotes Recovery of Neurological Function After Traumatic Brain Injury by Protecting the Neurovascular Unit. Transl Stroke Res 2020;11:720-33.

31. Lee L, Lo YT, See AAQ, et al. Long-term recovery profile of patients with severe disability or in vegetative states following severe primary intracerebral hemorrhage. J Crit Care 2018;48:269-75.

32. GBD 2016 Stroke Collaborators. Global, regional, and national burden of stroke, 1990-2016: a systematic analysis for the Global Burden of Disease Study 2016. Lancet Neurol 2019;18:439-58.

33. Li Q, Weiland A, Chen X, et al. Ultrastructural Characteristics of Neuronal Death and White Matter Injury in Mouse Brain Tissues After Intracerebral Hemorrhage: Coexistence of Ferroptosis, Autophagy, and Necrosis. Front Neurol 2018;9:581.

34. Xi G, Keep RF, Hoff JT. Mechanisms of brain injury after intracerebral haemorrhage. Lancet Neurol 2006;5:53-63.

35. Huan S, Jin J, Shi CX, et al. Overexpression of miR-146a inhibits the apoptosis of hippocampal neurons of rats with cerebral hemorrhage by regulating autophagy. Hum Exp Toxicol 2020;39:1178-89.

36. Ji W, Wang J, Xu J, et al. Lack of Aquaporin 9 Reduces Brain Angiogenesis and Exaggerates Neuronal Loss in the Hippocampus Following Intracranial Hemorrhage in Mice. J Mol Neurosci 2017;61:351-8.

37. Gong C, Hoff JT, Keep RF. Acute inflammatory reaction following experimental intracerebral hemorrhage in rat. Brain Res 2000;871:57-65.

38. Xue M, Del Bigio MR. Intracerebral injection of autologous whole blood in rats: time course of inflammation and cell death. Neurosci Lett 2000;283:230-2.

39. Duan X, Wen Z, Shen H, et al. Intracerebral Hemorrhage, Oxidative Stress, and Antioxidant Therapy. Oxid Med Cell Longev 2016;2016:1203285.

40. Li R, Yue J, Zhang Y, et al. CLOCK/BMAL1 regulates human nocturnin transcription through binding to the E-box of nocturnin promoter. Mol Cell Biochem 2008;317:169-77.

41. Hu J, Xue Y, Tang K, et al. The protective effects of hydrogen sulfide on the myocardial ischemia via regulating Bmal1. Biomed Pharmacother 2019;120:109540.

42. Liu WW, Wei SZ, Huang GD, et al. BMAL1 regulation of microglia-mediated neuroinflammation in MPTPinduced Parkinson's disease mouse model. FASEB J 2020;34:6570-81.

43. Bhatwadekar AD, Beli E, Diao Y, et al. Conditional Deletion of Bmal1 Accentuates Microvascular and Macrovascular Injury. Am J Pathol 2017;187:1426-35.

44. Zhang Y, Devocelle A, Souza L, et al. BMAL1 knockdown triggers different colon carcinoma cell fates by altering the delicate equilibrium between AKT/mTOR and P53/P21 pathways. Aging (Albany NY) 2020;12:8067-83.

45. Sloan MA, Price TR, Foulkes MA, et al. Circadian rhythmicity of stroke onset. Intracerebral and subarachnoid hemorrhage. Stroke 1992;23:1420-6.

46. Guan J, Ding Y, Liu Y, et al. Circadian effects on outcome following surgery for intracerebral hemorrhage in humans? Brain Res 2009;1258:78-85.

47. Saliminejad K, Khorram Khorshid HR, Soleymani Fard $\mathrm{S}$, et al. An overview of microRNAs: Biology, functions, therapeutics, and analysis methods. J Cell Physiol 2019;234:5451-65.

48. Bu Y, Yoshida A, Chitnis N, et al. A PERK-miR-211 axis suppresses circadian regulators and protein synthesis to promote cancer cell survival. Nat Cell Biol 2018;20:104-15.

49. Shende VR, Kim SM, Neuendorff N, et al. MicroRNAs function as cis- and trans-acting modulators of peripheral circadian clocks. FEBS Lett 2014;588:3015-22.

50. Onodera Y, Teramura T, Takehara T, et al. miR-155 induces ROS generation through downregulation of antioxidation-related genes in mesenchymal stem cells. Aging Cell 2017;16:1369-80.

51. Liang S, Hu J, Zhang A, et al. miR-155 induces endothelial cell apoptosis and inflammatory response in atherosclerosis by regulating Bmal1. Exp Ther Med 2020;20:128.

52. Zhang $W$, Li X, Tang $Y$, et al. miR-155-5p Implicates in the Pathogenesis of Renal Fibrosis via Targeting SOCS1 and SOCS6. Oxid Med Cell Longev 2020;2020:6263921. 
53. Eisenhardt SU, Weiss JB, Smolka C, et al. MicroRNA-155 aggravates ischemia-reperfusion injury by modulation of inflammatory cell recruitment and the respiratory oxidative burst. Basic Res Cardiol 2015;110:32.

54. Qu B, He J, Zeng Z, et al. MiR-155 inhibition alleviates suppression of osteoblastic differentiation by high glucose and free fatty acids in human bone marrow stromal cells by upregulating SIRT1. Pflugers Arch 2020;472:473-80.

55. Zhou Y, Wang S, Li Y, et al. SIRT1/PGC-1 $\alpha$ Signaling Promotes Mitochondrial Functional Recovery and Reduces Apoptosis after Intracerebral Hemorrhage in Rats. Front Mol Neurosci 2018;10:443.

56. Cheng Y, Zan J, Song Y, et al. Evaluation of intestinal injury, inflammatory response and oxidative stress following intracerebral hemorrhage in mice. Int $\mathrm{J}$ Mol Med 2018;42:2120-8.

57. Chen-Roetling J, Regan RF. Targeting the Nrf2-Heme Oxygenase-1 Axis after Intracerebral Hemorrhage. Curr Pharm Des 2017;23:2226-37.

58. Lee J, Moulik M, Fang Z, et al. Bmal1 and $\beta$-cell clock are

Cite this article as: Gong Y, Zhang G, Li B, Cao C, Cao D, Li X, Li H, Ye M, Shen H, Chen G. BMAL1 attenuates intracerebral hemorrhage-induced secondary brain injury in rats by regulating the Nrf2 signaling pathway. Ann Transl Med 2021;9(21):1617. doi: 10.21037/atm-21-1863 required for adaptation to circadian disruption, and their loss of function leads to oxidative stress-induced $\beta$-cell failure in mice. Mol Cell Biol 2013;33:2327-38.

59. Manfredini R, Boari B, Smolensky MH, et al. Circadian variation in stroke onset: identical temporal pattern in ischemic and hemorrhagic events. Chronobiol Int 2005;22:417-53.

60. Yao X, Wu B, Xu Y, et al. Day-night variability of hematoma expansion in patients with spontaneous intracerebral hemorrhage. J Biol Rhythms 2015;30:242-50.

61. Zheng D, Sato S, Cao YJ, et al. Circadian variation in clinical features and outcome of intracerebral hemorrhage: The INTERACT studies. Chronobiol Int 2016;33:1182-7.

62. Banks WA. From blood-brain barrier to blood-brain interface: new opportunities for CNS drug delivery. Nat Rev Drug Discov 2016;15:275-92.

63. Lo EH, Dalkara T, Moskowitz MA. Mechanisms, challenges and opportunities in stroke. Nat Rev Neurosci 2003;4:399-415. 


\section{Supplementary}

A

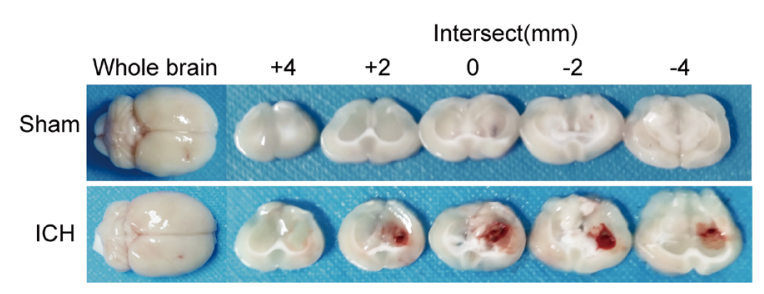

C

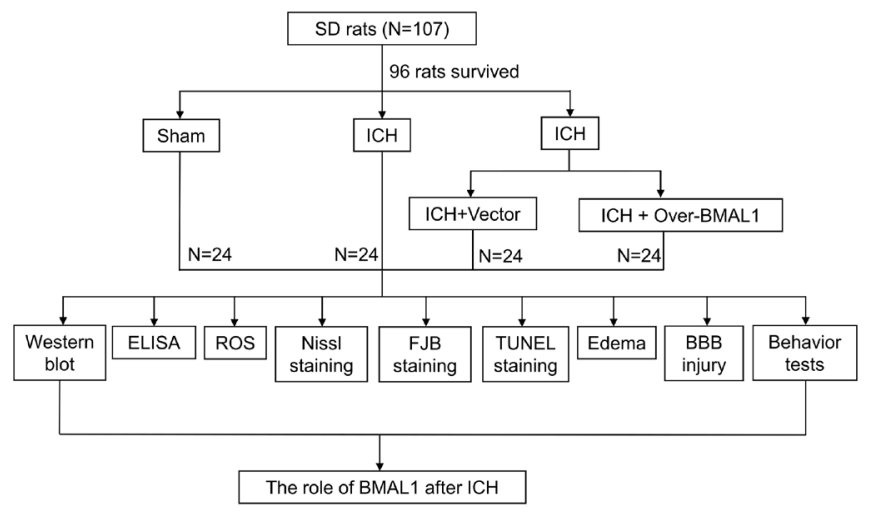

B

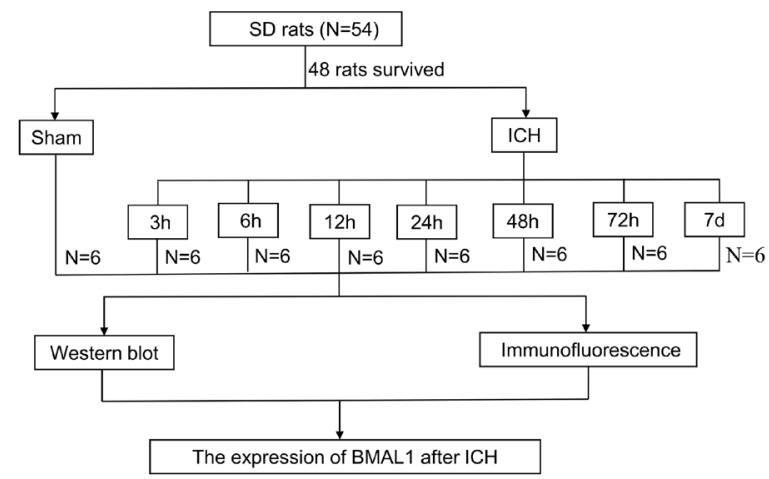

D

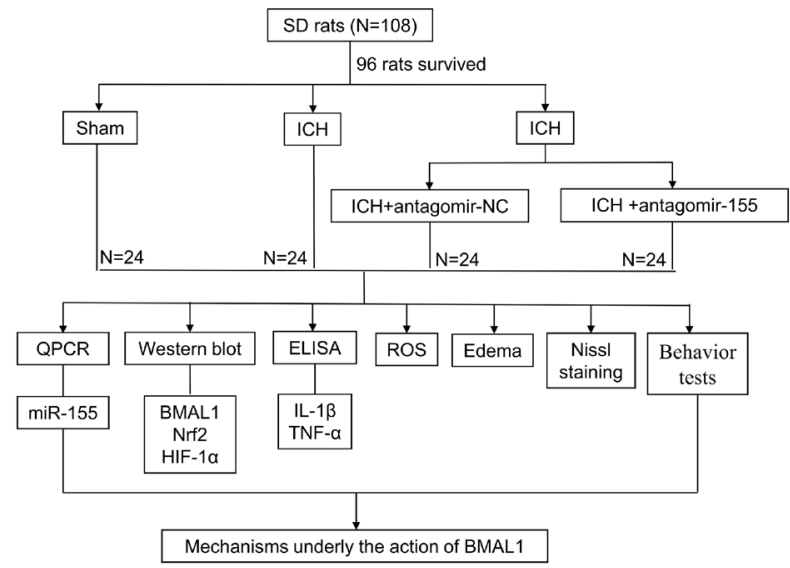

Figure S1 Intracerebral hemorrhage (ICH) model and experimental design. (A) Brain coronal sections obtained $24 \mathrm{~h}$ after the injection of autologous whole blood. (B) Experiment 1 was designed to assess the BMAL1 protein levels in the brain tissues of rats at various time points after ICH. (C) Experiment 2 was designed to evaluate the role of BMAL1 in brain injury induced by ICH. (D) Experiment 3 was designed to determine the effects of BMAL1 upregulation by inhibiting miR-155 and its underlying mechanisms on brain injury in rats. 